EUROPEAN ORGANIZATION FOR NUCLEAR RESEARCH

CERN-EP/2003-040

$14^{\text {th }}$ July 2003

\title{
Search for Anomalous Production of Di-lepton Events with Missing Transverse Momentum in $\mathrm{e}^{+} \mathrm{e}^{-}$Collisions at $\sqrt{s}=183-209 \mathrm{GeV}$
}

\author{
The OPAL Collaboration
}

\begin{abstract}
In total 1317 di-lepton events with significant missing transverse momentum were identified in a total data sample of $680 \mathrm{pb}^{-1}$ collected at $\mathrm{e}^{+} \mathrm{e}^{-}$centre-of-mass energies ranging from $183 \mathrm{GeV}$ to $209 \mathrm{GeV}$. The number of di-lepton events, the dependence on centreof-mass energy, and the event properties are consistent with expectations from Standard Model processes, predominantly $\mathrm{W}^{+} \mathrm{W}^{-}$production with both $\mathrm{W}$ bosons decaying leptonically. This topology is also an experimental signature for the pair production of new particles that decay to a charged lepton accompanied by one or more invisible particles. No evidence for new phenomena is apparent. Upper limits are presented on the production cross-section multiplied by the relevant branching ratio squared for sleptons, leptonically decaying charginos and charged Higgs bosons. Mass limits are also given.
\end{abstract}

(Submitted to Eur. Phys. J. C.) 


\section{The OPAL Collaboration}

G. Abbiendi ${ }^{2}$, C. Ainsley ${ }^{5}$, P.F. Åkesson ${ }^{3}$, G. Alexander ${ }^{22}$, J. Allison ${ }^{16}$, P. Amaral ${ }^{9}$, G. Anagnostou ${ }^{1}$, K.J. Anderson ${ }^{9}$, S. Arcelli ${ }^{2}$, S. Asai ${ }^{23}$, D. Axen ${ }^{27}$, G. Azuelos ${ }^{18, a}$, I. Bailey ${ }^{26}$, E. Barberio ${ }^{8, p}$, R.J. Barlow ${ }^{16}$, R.J. Batley ${ }^{5}$, P. Bechtle ${ }^{25}$, T. Behnke ${ }^{25}$, K.W. Bell ${ }^{20}$, P.J. Bell ${ }^{1}$,

G. Bella ${ }^{22}$, A. Bellerive ${ }^{6}$, G. Benelli ${ }^{4}$, S. Bethke ${ }^{32}$, O. Biebel ${ }^{31}$, O. Boeriu ${ }^{10}$, P. Bock $^{11}$,

M. Boutemeur ${ }^{31}$, S. Braibant ${ }^{8}$, L. Brigliadori' ${ }^{2}$, R.M. Brown ${ }^{20}$, K. Buesser ${ }^{25}$, H.J. Burckhart ${ }^{8}$,

S. Campana ${ }^{4}$, R.K. Carnegie ${ }^{6}$, B. Caron ${ }^{28}$, A.A. Carter ${ }^{13}$, J.R. Carter ${ }^{5}$, C.Y. Chang ${ }^{17}$,

D.G. Charlton ${ }^{1}$, A. Csilling ${ }^{29}$, M. Cuffiani ${ }^{2}$, S. Dado ${ }^{21}$, A. De Roeck ${ }^{8}$, E.A. De Wolf ${ }^{8, s}$,

K. Desch ${ }^{25}$, B. Dienes ${ }^{30}$, M. Donkers ${ }^{6}$, J. Dubbert ${ }^{31}$, E. Duchovni ${ }^{24}$, G. Duckeck ${ }^{31}$,

I.P. Duerdoth ${ }^{16}$, E. Etzion ${ }^{22}$, F. Fabbri ${ }^{2}$, L. Feld ${ }^{10}$, P. Ferrari ${ }^{8}$, F. Fiedler ${ }^{31}$, I. Fleck ${ }^{10}$, M. Ford ${ }^{5}$,

A. Frey ${ }^{8}$, A. Fürtjes ${ }^{8}$, P. Gagnon ${ }^{12}$, J.W. Gary ${ }^{4}$, G. Gaycken ${ }^{25}$, C. Geich-Gimbel ${ }^{3}$,

G. Giacomelli ${ }^{2}$, P. Giacomelli ${ }^{2}$, M. Giunta ${ }^{4}$, J. Goldberg ${ }^{21}$, E. Gross ${ }^{24}$, J. Grunhaus ${ }^{22}$,

M. Gruwé ${ }^{8}$, P.O. Günther ${ }^{3}$, A. Gupta ${ }^{9}$, C. Hajdu ${ }^{29}$, M.Hamann ${ }^{25}$, G.G. Hanson ${ }^{4}$, K. Harder ${ }^{25}$,

A. Harel ${ }^{21}$, M. Harin-Dirac ${ }^{4}$, M. Hauschild ${ }^{8}$, C.M. Hawkes ${ }^{1}$, R. Hawkings ${ }^{8}$, R.J. Hemingway ${ }^{6}$,

C. Hensel ${ }^{25}$, G. Herten ${ }^{10}$, R.D. Heuer ${ }^{25}$, J.C. Hill ${ }^{5}$, K. Hoffman ${ }^{9}$, D. Horváth ${ }^{29, c}$,

P. Igo-Kemenes ${ }^{11}$, K. Ishii ${ }^{23}$, H. Jeremie ${ }^{18}$, P. Jovanovic ${ }^{1}$, T.R. Junk ${ }^{6}$, N. Kanaya ${ }^{26}$, J. Kanzakii ${ }^{23, u}$, G. Karapetian ${ }^{18}$, D. Karlen ${ }^{26}$, K. Kawagoe ${ }^{23}$, T. Kawamoto ${ }^{23}$, R.K. Keeler ${ }^{26}$,

R.G. Kellogg ${ }^{17}$, B.W. Kennedy ${ }^{20}$, D.H. Kim ${ }^{19}$, K. Klein ${ }^{11, t}$, A. Klier ${ }^{24}$, S. Kluth ${ }^{32}$,

T. Kobayashi ${ }^{23}$, M. Kobel ${ }^{3}$, S. Komamiya ${ }^{23}$, L. Kormos ${ }^{26}$, T. Krämer ${ }^{25}$, P. Krieger ${ }^{6, l}$, J. von

Krogh $^{11}$, K. Kruger ${ }^{8}$, T. Kuhl ${ }^{25}$, M. Kupper ${ }^{24}$, G.D. Lafferty ${ }^{16}$, H. Landsman ${ }^{21}$, D. Lanske ${ }^{14}$,

J.G. Layter ${ }^{4}$, A. Leins ${ }^{31}$, D. Lellouch ${ }^{24}$, J. Letts ${ }^{o}$, L. Levinson ${ }^{24}$, J. Lillich ${ }^{10}$, S.L. Lloyd ${ }^{13}$,

F.K. Loebinger ${ }^{16}$, J. Lu ${ }^{27, w}$, J.Ludwig ${ }^{10}$, A. Macpherson ${ }^{28, i}$, W. Mader ${ }^{3}$, S. Marcellini' ${ }^{2}$,

A.J. Martin ${ }^{13}$, G. Masetti ${ }^{2}$, T. Mashimo ${ }^{23}$, P. Mättig ${ }^{m}$, W.J. McDonald ${ }^{28}$, J. McKenna ${ }^{27}$,

T.J. McMahon ${ }^{1}$, R.A. McPherson ${ }^{26}$, F. Meijers ${ }^{8}$, W. Menges ${ }^{25}$, F.S. Merritt ${ }^{9}$, H. Mes ${ }^{6, a}$,

A. Michelini ${ }^{2}$, S. Mihara ${ }^{23}$, G. Mikenberg ${ }^{24}$, D.J. Miller ${ }^{15}$, S. Moed ${ }^{21}$, W. Mohr ${ }^{10}$, T. Mori ${ }^{23}$,

A. Mutter ${ }^{10}$, K. Nagai ${ }^{13}$, I. Nakamura ${ }^{23, V}$, H. Nanjo ${ }^{23}$, H.A. Neal ${ }^{33}$, R. Nisius ${ }^{32}$, S.W. O'Neale ${ }^{1}$,

A. $\mathrm{Oh}^{8}$, A. Okpara ${ }^{11}$, M.J. Oreglia ${ }^{9}$, S. Orito ${ }^{23, *}$, C. Pahl ${ }^{32}$, G. Pásztor ${ }^{4, g}$, J.R. Pater ${ }^{16}$,

G.N.Patrick ${ }^{20}$, J.E. Pilcher ${ }^{9}$, J.Pinfold ${ }^{28}$, D.E. Plane ${ }^{8}$, B. Poli' ${ }^{2}$, J. Polok ${ }^{8}$, O. Pooth ${ }^{14}$,

M. Przybycieñ ${ }^{8, n}$, A. Quadt ${ }^{3}$, K. Rabbertz ${ }^{8, r}$, C. Rembser ${ }^{8}$, P. Renkel ${ }^{24}$, J.M. Roney ${ }^{26}$,

S. Rosati ${ }^{3}$, Y. Rozen ${ }^{21}$, K. Runge ${ }^{10}$, K. Sachs ${ }^{6}$, T. Saeki ${ }^{23}$, E.K.G. Sarkisyan ${ }^{8, j}$, A.D. Schaile ${ }^{31}$,

O. Schaile ${ }^{31}$, P. Scharff-Hansen ${ }^{8}$, J. Schieck ${ }^{32}$, T. Schörner-Sadenius ${ }^{8}$, M. Schröder ${ }^{8}$,

M. Schumacher ${ }^{3}$, C. Schwick ${ }^{8}$, W.G. Scott ${ }^{20}$, R. Seuster ${ }^{14, f}$, T.G. Shears ${ }^{8, h}$, B.C. Shen ${ }^{4}$,

P. Sherwood ${ }^{15}$, G. Siroli ${ }^{2}$, A. Skuja ${ }^{17}$, A.M. Smith ${ }^{8}$, R. Sobie ${ }^{26}$, S. Söldner-Rembold ${ }^{16, d}$,

F. Spano ${ }^{9}$, A. Stahl ${ }^{3}$, K. Stephens ${ }^{16}$, D. Strom ${ }^{19}$, R. Ströhmer ${ }^{31}$, S. Tarem ${ }^{21}$, M. Tasevsky ${ }^{8}$,

R.J. Taylor ${ }^{15}$, R. Teuscher ${ }^{9}$, M.A. Thomson ${ }^{5}$, E. Torrence ${ }^{19}$, D. Toya ${ }^{23}$, P. Tran ${ }^{4}$, I. Trigger ${ }^{8}$,

Z. Trócsányi ${ }^{30, e}$, E. Tsur ${ }^{22}$, M.F. Turner-Watson ${ }^{1}$, I. Ueda ${ }^{23}$, B. Ujvári' ${ }^{30, e}$, C.F. Vollmer ${ }^{31}$,

P. Vannerem ${ }^{10}$, R. Vértesi ${ }^{30}$, M. Verzocchi ${ }^{17}$, H. Voss ${ }^{8, q}$, J. Vossebeld ${ }^{8, h}$, D. Waller ${ }^{6}$, C.P. Ward ${ }^{5}$,

D.R. Ward ${ }^{5}$, P.M. Watkins ${ }^{1}$, A.T. Watson ${ }^{1}$, N.K. Watson ${ }^{1}$, P.S. Wells ${ }^{8}$, T. Wengler ${ }^{8}$,

N. Wermes ${ }^{3}$, D. Wetterling ${ }^{11}$ G.W.Wilson ${ }^{16, k}$, J.A. Wilson ${ }^{1}$, G. Wolf ${ }^{24}$, T.R. Wyatt ${ }^{16}$, S. Yamashita ${ }^{23}$, D. Zer-Zion ${ }^{4}$, L. Zivkovic ${ }^{24}$

${ }^{1}$ School of Physics and Astronomy, University of Birmingham, Birmingham B15 2TT, UK

${ }^{2}$ Dipartimento di Fisica dell' Università di Bologna and INFN, I-40126 Bologna, Italy 
${ }^{3}$ Physikalisches Institut, Universität Bonn, D-53115 Bonn, Germany

${ }^{4}$ Department of Physics, University of California, Riverside CA 92521, USA

${ }^{5}$ Cavendish Laboratory, Cambridge CB3 0HE, UK

${ }^{6}$ Ottawa-Carleton Institute for Physics, Department of Physics, Carleton University, Ottawa, Ontario K1S 5B6, Canada

${ }^{8}$ CERN, European Organisation for Nuclear Research, CH-1211 Geneva 23, Switzerland

${ }^{9}$ Enrico Fermi Institute and Department of Physics, University of Chicago, Chicago IL 60637, USA

${ }^{10}$ Fakultät für Physik, Albert-Ludwigs-Universität Freiburg, D-79104 Freiburg, Germany

${ }^{11}$ Physikalisches Institut, Universität Heidelberg, D-69120 Heidelberg, Germany

${ }^{12}$ Indiana University, Department of Physics, Bloomington IN 47405, USA

${ }^{13}$ Queen Mary and Westfield College, University of London, London E1 4NS, UK

${ }^{14}$ Technische Hochschule Aachen, III Physikalisches Institut, Sommerfeldstrasse 26-28, D-52056

Aachen, Germany

${ }^{15}$ University College London, London WC1E 6BT, UK

${ }^{16}$ Department of Physics, Schuster Laboratory, The University, Manchester M13 9PL, UK

${ }^{17}$ Department of Physics, University of Maryland, College Park, MD 20742, USA

${ }^{18}$ Laboratoire de Physique Nucléaire, Université de Montréal, Montréal, Québec H3C 3J7, Canada

${ }^{19}$ University of Oregon, Department of Physics, Eugene OR 97403, USA

${ }^{20}$ CLRC Rutherford Appleton Laboratory, Chilton, Didcot, Oxfordshire OX11 0QX, UK

${ }^{21}$ Department of Physics, Technion-Israel Institute of Technology, Haifa 32000, Israel

${ }^{22}$ Department of Physics and Astronomy, Tel Aviv University, Tel Aviv 69978, Israel

${ }^{23}$ International Centre for Elementary Particle Physics and Department of Physics, University of Tokyo, Tokyo 113-0033, and Kobe University, Kobe 657-8501, Japan

${ }^{24}$ Particle Physics Department, Weizmann Institute of Science, Rehovot 76100, Israel

${ }^{25}$ Universität Hamburg/DESY, Institut für Experimentalphysik, Notkestrasse 85, D-22607 Hamburg, Germany

${ }^{26}$ University of Victoria, Department of Physics, P O Box 3055, Victoria BC V8W 3P6, Canada

${ }^{27}$ University of British Columbia, Department of Physics, Vancouver BC V6T 1Z1, Canada

${ }^{28}$ University of Alberta, Department of Physics, Edmonton AB T6G 2J1, Canada

${ }^{29}$ Research Institute for Particle and Nuclear Physics, H-1525 Budapest, P O Box 49, Hungary

${ }^{30}$ Institute of Nuclear Research, H-4001 Debrecen, P O Box 51, Hungary

${ }^{31}$ Ludwig-Maximilians-Universität München, Sektion Physik, Am Coulombwall 1, D-85748

Garching, Germany

${ }^{32}$ Max-Planck-Institute für Physik, Föhringer Ring 6, D-80805 München, Germany

${ }^{33}$ Yale University, Department of Physics, New Haven, CT 06520, USA

${ }^{a}$ and at TRIUMF, Vancouver, Canada V6T $2 \mathrm{~A} 3$

${ }^{c}$ and Institute of Nuclear Research, Debrecen, Hungary

${ }^{d}$ and Heisenberg Fellow

${ }^{e}$ and Department of Experimental Physics, Lajos Kossuth University, Debrecen, Hungary

$f$ and MPI München

${ }^{g}$ and Research Institute for Particle and Nuclear Physics, Budapest, Hungary

${ }^{h}$ now at University of Liverpool, Dept of Physics, Liverpool L69 3BX, U.K.

${ }^{i}$ and CERN, EP Div, 1211 Geneva 23

$j$ and Manchester University 
${ }^{k}$ now at University of Kansas, Dept of Physics and Astronomy, Lawrence, KS 66045, U.S.A.

${ }^{l}$ now at University of Toronto, Dept of Physics, Toronto, Canada

$m$ current address Bergische Universität, Wuppertal, Germany

${ }^{n}$ now at University of Mining and Metallurgy, Cracow, Poland

${ }^{o}$ now at University of California, San Diego, U.S.A.

${ }^{p}$ now at Physics Dept Southern Methodist University, Dallas, TX 75275, U.S.A.

${ }^{q}$ now at IPHE Université de Lausanne, CH-1015 Lausanne, Switzerland

$r$ now at IEKP Universität Karlsruhe, Germany

${ }^{s}$ now at Universitaire Instelling Antwerpen, Physics Department, B-2610 Antwerpen, Belgium

${ }^{t}$ now at RWTH Aachen, Germany

${ }^{u}$ and High Energy Accelerator Research Organisation (KEK), Tsukuba, Ibaraki, Japan

$v$ now at University of Pennsylvania, Philadelphia, Pennsylvania, USA

w now at TRIUMF, Vancouver, Canada

* Deceased

\section{Introduction}

We report the final results from an investigation of events containing two oppositely charged leptons and significant missing transverse momentum recorded with the OPAL detector at LEP. These events were produced between 1997 and 2000 in the highest energy $\mathrm{e}^{+} \mathrm{e}^{-}$collisions achieved to date, at centre-of-mass energies $(\sqrt{s})$ ranging from 183 to $209 \mathrm{GeV}$. The data used in this analysis amount to a total integrated luminosity of $680 \mathrm{pb}^{-1}$. The event topology which is studied, henceforth called acoplanar di-lepton events, consists of low multiplicity events with two oppositely charged leptons, significant missing transverse momentum and the possible presence of additional photons.

The number of observed events and their properties are compared with the expectations for Standard Model processes, which are dominated by the $\ell^{+} \nu \ell^{-} \bar{\nu}$ final state $(\ell=\mathrm{e}, \mu, \tau)$ arising from $\mathrm{W}^{+} \mathrm{W}^{-}$production in which both $\mathrm{W}$ bosons decay leptonically. This topology is also an experimental signature for the pair production of new particles that decay to produce a charged lepton accompanied by one or more invisible particles. The invisible particles may be neutrinos, or the lightest stable supersymmetric [1] particle (LSP), or weakly interacting neutral particles with long lifetimes, which decay outside the detector volume. The LSP may be the lightest neutralino, $\tilde{\chi}_{1}^{0}$, the lightest sneutrino, $\tilde{\nu}$, or the gravitino, $\tilde{G}$. We present the results of searches for the pair production and stated decay mode of the following new particles:

charged scalar leptons (sleptons): $\tilde{\ell}^{ \pm} \rightarrow \ell^{ \pm} \tilde{\chi}_{1}^{0}\left(\right.$ or $\tilde{\ell}^{ \pm} \rightarrow \ell^{ \pm} \tilde{G}$ ), where $\tilde{\ell}^{ \pm}$may be a selectron $(\tilde{\mathrm{e}})$, smuon $(\tilde{\mu})$ or stau $(\tilde{\tau})$ and $\ell^{ \pm}$is the corresponding charged lepton.

charged Higgs: $\mathrm{H}^{ \pm} \rightarrow \tau^{ \pm} \nu_{\tau}$.

charginos: $\tilde{\chi}_{1}^{ \pm} \rightarrow \ell^{ \pm} \tilde{\nu}$ ("2-body" decays) or $\tilde{\chi}_{1}^{ \pm} \rightarrow \ell^{ \pm} \nu \tilde{\chi}_{1}^{0}$ ("3-body" decays).

The slepton searches are also relevant to interpreting the results of searches for chargino and neutralino production since the chargino and neutralino production cross-sections and branching ratios depend on the sneutrino and charged slepton masses. The search for charged sleptons provides constraints on the slepton masses, notably the selectron mass, while indirect 
limits on the sneutrino masses, notably the electron-sneutrino mass, can be obtained in models where the charged slepton and sneutrino masses are related. The search results described here will also be used in a separate publication regarding the search for sleptons with non-negligible lifetime.

In most respects the analysis is similar to our published searches at centre-of-mass energies of $161,172,183$ and $189 \mathrm{GeV}$ [2-4]. This paper supersedes the results of [3,4]. The analysis is performed in two stages. The first stage consists of a general event selection designed for all possible low multiplicity events containing a lepton pair plus missing transverse momentum (Section 3). In this context the Standard Model $\ell^{+} \nu \ell^{-} \bar{\nu}$ events are considered as signal in addition to possible new physics sources. All Standard Model processes that do not lead to $\ell^{+} \nu \ell^{-} \bar{\nu}$ final states, e.g. $\mathrm{e}^{+} \mathrm{e}^{-} \ell^{+} \ell^{-}$and $\ell^{+} \ell^{-}(\gamma)$, are considered as background and are reduced to a rather low level $(\approx 3 \%)$ by the event selection. The observed numbers of events and kinematic distributions for the data are compared with expectations from Standard Model processes in Section 4. In the second stage the detailed properties of the events (e.g. the type of leptons observed and their momenta), which vary greatly depending on the type of new particles considered and on free parameters within the models, are used to separate as far as possible the events consistent with potential new physics sources from $\mathrm{W}^{+} \mathrm{W}^{-}$production and other Standard Model processes (Section 5). Constraints on new physics are discussed in Section 6 .

Slepton search results from the ALEPH collaboration at $\sqrt{s} \leq 209 \mathrm{GeV}$ [5] have been published recently. The L3 and DELPHI collaborations have published searches for sleptons in this channel using data with $\sqrt{s} \leq 189 \mathrm{GeV}[6]$.

\section{OPAL Detector and Monte Carlo Simulation}

A detailed description of the OPAL detector can be found elsewhere $[7,8]$ and only the general features are described here.

The central detector consisted of a system of chambers providing charged particle tracking over $96 \%$ of the full solid angle inside a $0.435 \mathrm{~T}$ uniform magnetic field parallel to the beam axis. It consisted of a two-layer silicon micro-strip vertex detector, a high precision vertex drift chamber, a large volume jet chamber and a set of $z$-chambers that measured the track coordinates along the beam direction.

A lead-glass electromagnetic calorimeter (ECAL) was located outside the magnet coil. It provided, in combination with the gamma-catchers (GC) and forward detectors (FD), which were lead-scintillator sandwich calorimeters and, at smaller angles, silicon tungsten calorimeters (SW), geometrical acceptance with excellent hermeticity down to approximately $25 \mathrm{mrad}^{1}$ from the beam direction.

The magnet return yoke was instrumented for hadron calorimetry and consisted of barrel and endcap sections along with pole tip detectors that together covered the region $|\cos \theta|<0.99$. Outside the hadron calorimeter (HCAL), four layers of muon chambers covered the polar angle range of $|\cos \theta|<0.98$. Arrays of thin scintillating tiles were installed in the endcap region

\footnotetext{
${ }^{1}$ For some polar angles, precision energy measurements were compromised by upstream material such as the synchrotron shield installed for LEP2 at approximately $30 \mathrm{mrad}$. However, the ability to veto significant energy deposits with very high efficiency extended down to polar angles of $25 \mathrm{mrad}$.
} 
to improve trigger performance, time resolution and hermeticity for operation at LEP 2 [8]. Of particular relevance to this analysis are the four layers of scintillating tiles (the MIP-plug) installed at each end of the OPAL detector covering the angular range $43<\theta<220 \mathrm{mrad}$. These tiles were commissioned in 1997 and became fully operational for data taken from 1998 at $\sqrt{s} \geq 189 \mathrm{GeV}$. Time-of-flight (TOF) scintillators in the barrel region aided cosmic ray rejection.

The following Standard Model processes are simulated. Four-fermion production is simulated using the KORALW [9] generator which uses the grc4f [10] matrix elements to calculate the four-fermion cross-sections including interference effects and includes a detailed description of hard radiation from initial and final state charged particles. Two-photon production of muon pairs and tau pairs is generated with the BDK generator [11] and the program of Vermaseren [12] is used for the two-photon production of electron pairs. The grc4f event generator excluding multi-peripheral processes is used for $\mathrm{e}^{+} \mathrm{e}^{-} \ell^{+} \ell^{-}$and $\mathrm{e}^{+} \mathrm{e}^{-} \mathrm{q} \overline{\mathrm{q}}$. The PhoJEt [13] and HERWIG [14] event generators are used to study backgrounds from two-photon production of hadrons. The production of lepton pairs is generated using BHwIDE [15] and TEEGG [16] for $\mathrm{e}^{+} \mathrm{e}^{-}(\gamma)$, and KK2F [17] for $\mu^{+} \mu^{-}(\gamma)$ and $\tau^{+} \tau^{-}(\gamma)$. The final state $\nu \bar{\nu} \gamma(\gamma)$ is generated with NunugPV98 [18].

Slepton pair production is generated using Susygen [19]. Chargino pair production is generated using DFGT [20] for three-body decays, and SuSYGEN for two-body decays. Charged Higgs boson pair production is generated using HzHA [21].

Backgrounds from the accelerator or cosmic-ray interactions can lead to additional hits, energy deposition and even reconstructed tracks being superimposed on triggered data events. Such effects of detector occupancy are simulated for all Monte Carlo samples by adding to the Monte Carlo events the hits, energy depositions and additional jets found in randomly triggered beam-crossing data events (BXRSA trigger [22] with a constant $0.1 \mathrm{~Hz}$ rate) corresponding to the same centre-of-mass energy. Systematic checks showed that this constant rate model adequately described the average inefficiency during a LEP fill, despite the instantaneous luminosity decreasing typically by a factor of 2 or 3 .

All Standard Model and new physics Monte Carlo samples are processed with a full simulation of the OPAL detector [23] and subjected to the same reconstruction and analysis programs as used for the OPAL data.

\section{General Event Selection}

\subsection{Introduction}

The general event selection is formed by requiring that an event be selected by either or both of two independent event selections, referred to here as selection I and selection II. Selection I is designed to retain efficiency for events with low visible energy. This is characteristic of slepton or chargino events where the mass difference, $\Delta m$, between the parent particle and the invisible daughter particle (e.g. $\tilde{\chi}_{1}^{0}$ ) is small. Selection II is optimised to maximise the efficiency for $\mathrm{W}^{+} \mathrm{W}^{-} \rightarrow \ell^{+} \nu \ell^{-} \bar{\nu}$ events, while keeping other Standard Model background events to a minimum. 
Both selections require evidence for two ${ }^{2}$ charged leptons and an invisible system carrying significant missing transverse momentum $\left(p_{\mathrm{t}}^{\text {miss }}\right)$. The maximum $p_{\mathrm{t}}^{\text {miss }}$ which can be carried away by undetected particles travelling close to the beam is set by the maximum angle to the beam at which such a particle will not be detected. This is $25 \mathrm{mrad}$ - the angle to which the silicon tungsten detector extends. A particle with energy of $E_{\text {beam }}$ may thus carry away $p_{\mathrm{t}}^{\text {miss }}=0.025 E_{\text {beam }}$ without being detected.

Some background processes containing secondary neutrinos (particularly from tau decay) may have large values of $p_{\mathrm{t}}^{\text {miss }}$ with the direction of the missing momentum vector pointing away from the beam axis. Such events tend to be fairly coplanar, and the component of $p_{\mathrm{t}}^{\text {miss }}$ which is perpendicular to the event thrust axis in the transverse plane (called $a_{\mathrm{t}}^{\text {miss }}$ ) is much less sensitive than $p_{\mathrm{t}}^{\text {miss }}$ to the presence of neutrinos from tau decays or to poorly measured particles. This can be seen by considering electrons produced in tau decay. Low momentum electrons produced from this source can have a large angle relative to the original tau direction, but their momentum transverse to the original direction (and hence their contribution to $a_{\mathrm{t}}^{\text {miss }}$ ) is small.

The event selection as it was first implemented was described in detail in [2]. In [3] we made use of the improved hermeticity for non-showering particles in the forward direction provided by the MIP-plug ${ }^{3}$. Subsequent improvements have been made for the analysis of the data taken at $189 \mathrm{GeV}$ [4] by reducing the sensitivity to mis-measurements of $p_{\mathrm{t}}^{\text {miss }}$ and using the much improved background rejection capabilities of the MIP-plug to increase substantially the efficiency of selection II. For this paper, selection I has been consolidated and much refined. In particular, a selection has been added for single lepton events in which a single high transverse momentum charged lepton is observed in the central detector. These are usually events where the second charged lepton is produced at a polar angle sufficiently close to the beam direction that it does not produce a visible track in the central detector. In the context of this paper, this "single-lepton" selection is used to recuperate di-leptons where the second lepton was missed. Various small modifications were made to selection II in order to reduce sensitivity to poorly modelled backgrounds. An example of this is using stricter track quality criteria to reduce the possibility of tracks from overlaid beam-gas events affecting the measurement of the event kinematics.

The current event selection is summarised below.

\subsection{Selection I}

Selection I is designed to retain efficiency for events with very low visible energy, but nevertheless significant $p_{\mathrm{t}}^{\text {miss. }}$. This is typical of new physics signal events with small $\Delta m$. The selection requires evidence that a pair of leptons has been produced and of significant missing transverse momentum. Subsequent cuts reduce the probability that the signature of missing transverse momentum is faked by events with secondary neutrinos from tau decay or poorly measured particles.

\footnotetext{
${ }^{2}$ This is not strictly true for the single lepton selection of selection I (Section 3.2.3).

${ }^{3}$ Note that the general event selection and the Monte Carlo simulation samples used for the $183 \mathrm{GeV}$ dataset are unchanged from the $183 \mathrm{GeV}$ publication. The poorer MIP-plug detector performance in 1997 would have necessitated a re-optimisation of the latest general event selection, and we judged such a re-analysis to be inappropriate.
} 
At least one lepton in the event is required to be well identified and to satisfy requirements on isolation and transverse momentum. Much looser requirements are made on the possible presence of a second lepton in the event.

In order to be considered in the event selection, tracks in the central detector are required to satisfy $p_{t}>0.1 \mathrm{GeV}$. Clusters in the barrel ECAL are required to satisfy $E>0.1 \mathrm{GeV}$ and clusters in the endcap ECAL are required to satisfy $E>0.2 \mathrm{GeV}$. Converting photons are identified and the tracks and clusters associated to the conversion are replaced by a single 4 -vector representing the photon.

\subsubsection{Lepton Candidates}

The first stage is to look for lepton candidates in the event. A track is identified as a lepton candidate if it has $p>1.5 \mathrm{GeV}$ and it is identified as an electron, muon or hadronic tau decay ${ }^{4}$. The electron ID is based on the ratio of ECAL energy to track momentum $(E / p)$, and $\mathrm{d} E / \mathrm{d} x$ information. Muons are identified using muon chamber or HCAL hits which match with a track in the central detector, or from a high momentum track which matches with a low energy ECAL cluster. To identify a hadronic tau the following criteria are applied:

- Within a cone of half-opening angle $35^{\circ}$ around a track of $p_{t}>1.5 \mathrm{GeV}$ there are no more than three tracks in total.

- The invariant mass of all tracks and clusters within the cone is less than the tau mass. This is calculated assuming the pion mass for each track and correcting for double counting of tracks and clusters.

The lepton candidates are also required to be isolated. There must be no more than 2 additional tracks and no more than 2 additional clusters in an isolation cone defined around the lepton candidate (half opening angle $20^{\circ}$ for electrons and muons, half opening angle $60^{\circ}$ for hadronic tau decays), and the energy sum of the tracks or of the clusters must be less than $2 \mathrm{GeV}$.

\subsubsection{Di-lepton Event Selection}

Firstly evidence for a pair of leptons is required:

- There must be at least one and no more than two isolated lepton candidates with $p_{\mathrm{t}}>$ $1.5 \mathrm{GeV}$.

- If the event contains two isolated lepton candidates then all tracks in the event must be associated with the lepton candidates.

- If there is only one isolated lepton candidate then the other tracks and clusters in the event are considered as a possible second lepton candidate provided the following:

\footnotetext{
${ }^{4}$ The lepton identification (ID) made at the event selection stage is used for the purpose of the selection only. A separate lepton ID is applied to selected events and used by the search analysis (see section 4.1).
} 
(a) There must be between 1 and 3 additional tracks, at least one of which must have $p_{\mathrm{t}}>0.3 \mathrm{GeV}$.

(b) The invariant mass of the additional tracks must be less than $3 \mathrm{GeV}$ and the invariant mass of the additional tracks and clusters must be less than $8 \mathrm{GeV}$.

(c) $\gamma \beta$, the net momentum of the additional tracks and clusters divided by their invariant mass, is required to be greater than 2.0.

Next, significant missing transverse momentum is required. For events with a large acoplanarity angle ( $\phi_{\text {acop }}>\pi / 2$ where $\phi_{\text {acop }}$ is the supplement of the azimuthal opening angle), the following cut is applied ${ }^{5}$ :

- $x_{\mathrm{t}}>0.045$, where $x_{\mathrm{t}}$ is the scaled missing transverse momentum of the event $\left(p_{\mathrm{t}}^{\text {miss }} / E_{\mathrm{beam}}\right)$.

For events with small acoplanarity angle $\left(\phi_{\text {acop }}<\pi / 2\right)$ :

- $x_{\mathrm{t}}>0.035$

- At small acoplanarity, cuts on $a_{\mathrm{t}}^{\text {miss }} / E_{\mathrm{beam}}$ and $\theta_{\mathrm{a}}^{\text {miss }}$ (where $\theta_{\mathrm{a}}^{\text {miss }}=\tan ^{-1}\left[a_{\mathrm{t}}^{\text {miss }} / p_{\mathrm{z}}^{\text {miss }}\right]$, and $p_{\mathrm{z}}^{\text {miss }}$ is the total momentum of the observed particles in the $\mathrm{z}$ direction) are used to reduce background from processes such as $\tau^{+} \tau^{-}$and $\mathrm{e}^{+} \mathrm{e}^{-} \tau^{+} \tau^{-}$. Events are divided into subsets using variables which help estimate how likely they are to originate from $\tau^{+} \tau^{-}$or $\mathrm{e}^{+} \mathrm{e}^{-}$ $\tau^{+} \tau^{-}$. The cut values vary according to the subset (in increasing order of similarity to $\tau^{+} \tau^{-}$or $\mathrm{e}^{+} \mathrm{e}^{-} \tau^{+} \tau^{-}$background):

(a) $x_{\mathrm{t}}>0.2$ and unassociated energy less than $1.5 \mathrm{GeV}$ and the lepton pair is identified as $\mathrm{e}^{+} \mathrm{e}^{-}, \mu^{+} \mu^{-}$or $\mathrm{e}^{ \pm} \mu^{\mp}: a_{\mathrm{t}}^{\text {miss }} / E_{\text {beam }}>0.011$ and $\theta_{\mathrm{a}}^{\text {miss }}>0.025$

(b) $x_{\mathrm{t}}>0.2$ or unassociated energy less than $1.5 \mathrm{GeV}$ but not in subset (a) $: a_{\mathrm{t}}^{\text {miss }} / E_{\text {beam }}>$ 0.018 and $\theta_{\mathrm{a}}^{\mathrm{miss}}>0.05$

(c) $x_{\mathrm{t}}<0.2$ and unassociated energy greater than $1.5 \mathrm{GeV}: a_{\mathrm{t}}^{\text {miss }} / E_{\text {beam }}>0.025$ and $\theta_{\mathrm{a}}^{\text {miss }}>0.1$

- $x_{\mathrm{t}}+a_{\mathrm{t}}^{\text {miss }} / E_{\mathrm{beam}}>0.07$.

Further cuts are applied to reduce the effect of processes which may fake the signature of missing transverse momentum. These are mostly vetoes against energy in the forward region (GC, FD, SW or MIP-plug). However care is taken to ensure that the activity in the forward region is only used to veto the event if the activity could possibly explain the apparent missing momentum. Also different requirements are made depending on the amount of missing transverse momentum observed.

Radiative lepton pair events containing high energy isolated photons form a potentially serious source of background, because quantities such as $p_{\mathrm{t}}^{\text {miss }}$ and $a_{\mathrm{t}}^{\text {miss }}$ may be poorly measured. However, in order to retain efficiency for potential signal events containing isolated photons, events are rejected only if the presence of the isolated photon could possibly have caused the observed $p_{\mathrm{t}}^{\text {miss }}$ and $a_{\mathrm{t}}^{\text {miss }}$.

\footnotetext{
${ }^{5}$ The effect of measurement errors is taken into account in selection I by taking each lepton in turn and shifting its momentum up and down by one standard deviation of its estimated measurement error. At each stage the values of $p_{\mathrm{t}}^{\text {miss }}$ and $a_{\mathrm{t}}^{\text {miss }}$ are recalculated and the minimum of all the values obtained is the one used for comparison to the cut values.
} 


\subsubsection{Single Lepton Selection}

Selection I also includes a selection for events with only one lepton visible in the central detector, and the other lepton travelling sufficiently close to the beam axis that no track is produced in the central tracking chambers.

The principal single lepton selection criteria are:

- The event must contain one and only one identified, isolated lepton candidate, and no other tracks.

- $x_{\mathrm{t}}>0.16$

In order to veto events which may fake the missing transverse momentum signature, events are rejected if they contain activity in ECAL, GC, FD, SW, muon endcap or MIP-plug which is back-to-back with the observed lepton.

\subsection{Selection II}

Selection II is optimised to select high visible energy events typical of the $\mathrm{W}^{+} \mathrm{W}^{-} \rightarrow \ell^{+} \nu \ell^{-} \bar{\nu}$ process. A low multiplicity preselection is applied such that the events contain at least one track but no more than 8 . Also the sum of the number of tracks plus the number of ECAL clusters is required to be less than 16 .

A cone-based jet finding algorithm [24] is applied requiring a minimum jet energy of $2.5 \mathrm{GeV}$ and a cone half angle of $20^{\circ}$. Events are required to contain 1, 2 or 3 jets, and a separate selection is used for each value of $n_{\text {jet }}$. The majority (about 90\%) of $\mathrm{W}^{+} \mathrm{W}^{-} \rightarrow \ell^{+} \nu \ell^{-} \bar{\nu}$ events have $n_{\text {jet }}=2$. One-jet events are usually those where the decay products from one of the $\mathrm{W}$ bosons are poorly reconstructed (for example if the lepton is travelling close to the beam pipe). Three-jet events can occur if there is an energetic photon in the event.

Electron and muon identification, similar to that in selection I, is applied to the most energetic track in each jet. Jets not identified as electrons or muons are classified as hadronic tau decays.

The most important cuts for each $n_{\text {jet }}$ class are summarised below.

\subsubsection{Di-jet Selection}

1. $\theta_{\text {acol }}>5^{\circ}$, where $\theta_{\text {acol }}$ is the acolinearity angle between the two jets (defined as the supplement of the three-dimensional opening angle).

2. $x_{\mathrm{t}}>0.05$. It is further required that the significance by which $x_{\mathrm{t}}$ exceeds 0.05 be greater than 1 standard deviation.

3. For events with $\phi_{\text {acop }}>\pi / 2$ it is required that the direction of the missing momentum satisfy $\left|\cos \theta_{p}^{\text {miss }}\right|<0.95$. For events with $\phi_{\text {acop }}<\pi / 2$ it is required that $a_{\mathrm{t}}^{\text {miss }} / E_{\text {beam }}>$ 0.022 and that $\sin \theta_{\mathrm{a}}^{\mathrm{miss}}>0.06$. 
Further cuts are made on the quality of each jet, and for background rejection (mainly for events at low $\left.x_{\mathrm{t}}\right)$ :

- Events are rejected if there are any tracks which are not associated with either jet.

- Events with low $x_{\mathrm{t}}\left(x_{\mathrm{t}}<0.15\right)$ are rejected if there is evidence of activity in the MIPplug with azimuthal angle within $60^{\circ}$ of the missing transverse momentum direction. For events with medium $x_{\mathrm{t}}\left(0.15<x_{\mathrm{t}}<0.23\right)$, the veto uses only the outer MIP-plug scintillators.

\subsubsection{Tri-jet Selection}

For events classified as tri-jet, significant missing momentum is required:

- The sum of the opening angles among the three two-jet pairings should be less than $359^{\circ}$.

- $x_{\mathrm{t}}$ of the three-jet system should exceed 0.05 with a significance exceeding 1 standard deviation.

Further requirements depend in part on the three-jet event topology in the transverse plane characterised by $\Delta \phi^{\max }$. This is calculated by ordering the jets by increasing azimuth, and finding the maximum azimuthal di-jet separation angle when rotating clockwise from one jet to the next ${ }^{6}$.

The most important additional requirements are:

- For $\Delta \phi^{\max }>185^{\circ}$, there should be no pair of vertex drift chamber axial tracks with an azimuthal opening angle exceeding $165^{\circ}$. These tracks are reconstructed independently from standard jet chamber based tracks and this criterion helps to reduce cases where the wrong jet chamber left-right ambiguity is chosen in the standard tracking.

- For $\Delta \phi^{\max }<185^{\circ}$ it is explicitly required that two of the jets have associated tracks (charged jet) and the third jet has no associated tracks (neutral jet). For the events with $\Delta \phi^{\max }<180^{\circ}$ an axis in the transverse plane is defined using the most energetic charged jet. The event is rejected if the transverse momentum of the neutral jet with respect to this axis exceeds $80 \%$ of the transverse momentum of the less energetic charged jet. This cut is effective against $\tau^{+} \tau^{-} \gamma$ events.

- Events are rejected if there is evidence for a particle passing through the MIP-plug (similar to di-jet MIP-plug veto but without the directional requirement).

\subsubsection{Single-jet Selection}

The single-jet selection applies to events where one lepton with high transverse momentum is observed at wide angle $|\cos \theta|<0.82$ with evidence for a partially reconstructed lepton at small polar angle, or events where the two leptons fall within the same cone (massive mono-jet)

\footnotetext{
${ }^{6}$ This definition results in $\Delta \phi^{\text {max }}$ being in the range from $120^{\circ}$ to $360^{\circ}$.
} 
for $|\cos \theta|<0.90$. In contrast to the single lepton category of selection I, it is required here that there be some activity in the forward region (endcap ECAL, GC, FD, SW, muon endcap, MIP-plug) when there is evidence for only one lepton at wide angle.

Backgrounds from cosmic rays are reduced by requiring in-time TOF hits (within $20 \mathrm{ns)} \mathrm{for}$ tracks in the barrel region, and that the most energetic track in the jet be associated with hits in the silicon micro-vertex detector.

Three different minimum requirements on $x_{\mathrm{t}}$ are applied $(0.20,0.30$ and 0.25$)$ depending on whether the event satisfies the massive mono-jet criteria and whether the forward activity is solely due to the MIP-plug or not, respectively.

\section{Comparison of Data with Standard Model Processes}

The numbers of events passing the general selection at each centre-of-mass energy in the data are compared with the Standard Model Monte Carlo predictions in Table 1. The total number of events predicted by the Standard Model is given, together with a breakdown into the contributions from individual processes. The data have been binned by centre-of-mass energy in bins which reflect the predominant centre-of-mass energies where data was collected. The number of observed candidates is consistent with the expectation from Standard Model sources, which is dominated by the $\ell^{+} \nu \ell^{-} \bar{\nu}$ final state arising mostly from $\mathrm{W}^{+} \mathrm{W}^{-}$production.

\begin{tabular}{|rr|rr|rrrrr|}
\hline$\sqrt{s}$ & $\mathcal{L}$ & Data & $\mathrm{SM}$ & $\ell^{+} \nu \ell^{-} \bar{\nu}$ & $\mathrm{e}^{+} \mathrm{e}^{-} \ell^{+} \ell^{-}$ & $\ell \ell \mathrm{q} \overline{\mathrm{q}}$ & $\ell^{+} \ell^{-}(\gamma)$ & $\nu \bar{\nu} \gamma(\gamma)$ \\
\hline 182.7 & 56.4 & 78 & $81.4 \pm 0.8$ & $77.5 \pm 0.7$ & $3.4 \pm 0.5$ & $0.07 \pm 0.03$ & $0.31 \pm 0.04$ & $0.06 \pm 0.03$ \\
188.6 & 183.5 & 332 & $348.2 \pm 1.9$ & $337.2 \pm 1.7$ & $3.6 \pm 0.7$ & $1.6 \pm 0.1$ & $4.6 \pm 0.3$ & $1.1 \pm 0.2$ \\
191.6 & 29.3 & 60 & $56.1 \pm 0.6$ & $54.2 \pm 0.6$ & $0.6 \pm 0.1$ & $0.2 \pm 0.04$ & $0.9 \pm 0.2$ & $0.10 \pm 0.04$ \\
195.5 & 76.4 & 166 & $150.5 \pm 1.2$ & $144.7 \pm 1.0$ & $2.7 \pm 0.4$ & $0.6 \pm 0.06$ & $2.1 \pm 0.4$ & $0.42 \pm 0.05$ \\
199.5 & 76.6 & 155 & $153.5 \pm 0.9$ & $148.7 \pm 0.7$ & $1.8 \pm 0.3$ & $0.7 \pm 0.06$ & $1.8 \pm 0.2$ & $0.45 \pm 0.05$ \\
202.0 & 45.5 & 110 & $90.6 \pm 0.7$ & $87.6 \pm 0.6$ & $1.4 \pm 0.2$ & $0.4 \pm 0.04$ & $1.0 \pm 0.1$ & $0.19 \pm 0.04$ \\
205.1 & 79.0 & 154 & $155.6 \pm 1.2$ & $150.8 \pm 1.1$ & $1.8 \pm 0.3$ & $0.6 \pm 0.07$ & $2.0 \pm 0.3$ & $0.38 \pm 0.07$ \\
206.5 & 124.6 & 243 & $249.5 \pm 1.4$ & $241.4 \pm 1.2$ & $3.9 \pm 0.6$ & $1.1 \pm 0.1$ & $2.3 \pm 0.3$ & $0.64 \pm 0.08$ \\
207.9 & 9.03 & 19 & $18.2 \pm 0.2$ & $17.7 \pm 0.2$ & $0.20 \pm 0.04$ & $0.08 \pm 0.01$ & $0.18 \pm 0.03$ & $0.06 \pm 0.01$ \\
\hline All & 680.4 & 1317 & $1303.6 \pm 3.3$ & $1259.8 \pm 2.9$ & $19.6 \pm 1.2$ & $5.4 \pm 0.2$ & $15.2 \pm 0.7$ & $3.4 \pm 0.2$ \\
\hline
\end{tabular}

Table 1: Comparison between data and Monte Carlo of the number of events passing the general selection in each centre-of-mass energy bin. The total number of events predicted by the Standard Model is given, together with a breakdown into the contributions from individual processes. The Monte Carlo statistical errors are shown. Also listed for each bin is the mean $\sqrt{s}$ (in $G e V$ ) and the integrated luminosity, $\mathcal{L}$, in $p b^{-1}$.

\subsection{Lepton Identification and Kinematic Distributions}

Information about the type of lepton found in the selected events and measurement of the event kinematics was used to compare the data with expectations from Standard Model processes. Some of these distributions are then used as likelihood variables to distinguish between Standard Model and new physics sources of acoplanar di-lepton events (section 5). We examine the 


\begin{tabular}{|c|c|c|c|}
\hline \multirow{2}{*}{ Lepton ID } & \multicolumn{3}{|c|}{ True Identity of Lepton } \\
\cline { 2 - 4 } & $\mathrm{e}$ or $\tau \rightarrow \mathrm{e}$ & $\mu$ or $\tau \rightarrow \mu$ & $\tau \rightarrow \mathrm{h}$ \\
\hline $\mathrm{e}$ & $96.3 \%$ & $0.1 \%$ & $4.7 \%$ \\
$\mu$ & $0.1 \%$ & $98.2 \%$ & $3.5 \%$ \\
$\mathrm{~h}$ & $1.2 \%$ & $0.8 \%$ & $86.9 \%$ \\
$\mathrm{e} / \mathrm{h}$ & $2.2 \%$ & $0.1 \%$ & $2.8 \%$ \\
$\mu / \mathrm{h}$ & $0.2 \%$ & $0.8 \%$ & $1.8 \%$ \\
$\mathrm{x}$ & $0.0 \%$ & $0.0 \%$ & $0.4 \%$ \\
\hline
\end{tabular}

Table 2: Lepton identification performance, calculated using KoRALW four-lepton Monte Carlo events at $\sqrt{s}=189-206 \mathrm{GeV}$. The efficiency is evaluated for leptons generated with $|\cos \theta|<0.95$ and $p / E_{\text {beam }}>0.02$.

distributions of the likelihood variables and other related kinematics distributions, as measured in the data, and compare to expectations from Standard Model processes. The following distributions are used as the likelihood variables:

- Di-lepton identities;

- Acolinearity of the event, defined as the supplement of the three-dimensional opening angle between the two leptons;

- Momentum asymmetry of the event, $\left(\left|p_{1}-p_{2}\right| /\left(p_{1}+p_{2}\right)\right)$, where $p_{1}$ and $p_{2}$ are the momenta of each lepton;

- Scaled momentum of each lepton, $\left(p / E_{\text {beam }}\right)$ using the best estimate combining tracking and calorimetry information;

- $-q \cos \theta$ of each lepton, where $q$ is the particle charge and $\theta$ is the polar angle with respect to the electron beam direction.

The lepton identification algorithm has been improved using techniques developed for tau lepton decay analyses such as described in [25]. The efficiencies for correctly identifying leptons as electrons, muons or hadronically decaying tau leptons are shown in Table 2 for a sample of leptons representative of the geometrical and kinematical acceptance of the general selection. Note that we have six separate mutually exclusive lepton identification decisions: electron (e), muon $(\mu)$, hadronically decaying tau (h), electron-hadron ambiguous (e/h), muon-hadron ambiguous $(\mu / \mathrm{h})$ and "rest-of-event" $(\mathrm{x})$. The ambiguous categories correspond to cases where the lepton candidate has properties which do not permit clear identification between the two lepton types, while the rest-of-event category arises mostly from tau leptons which fail to pass basic lepton identification criteria.

We show in Figure 1 the observed di-lepton identities and the above kinematic variables are shown in Figure 2. Additional kinematic variables, which are not directly used in the likelihood, are also shown in Figure 3. Reasonable agreement is observed between the data and the Standard Model Monte Carlo in all these distributions as quantified using $\chi^{2}$ tests. 


\section{Likelihood Method for New Physics Search}

\subsection{Method}

The method used in the second stage of the analysis, in which we distinguish between Standard Model and new physics sources of lepton pair events with missing momentum, is essentially described in [4]. Discrimination is provided by information on the lepton identification, the acolinearity and momentum asymmetry of the event, the scaled momentum and $-q \cos \theta$ of the observed lepton candidates. These variables are combined using a likelihood technique. The discriminating quantity used is the relative likelihood, $L_{\mathrm{R}}$, defined by:

$$
L_{\mathrm{R}}=\frac{L_{\mathrm{S}}}{L_{\mathrm{S}}+L_{\mathrm{B}}},
$$

where $L_{\mathrm{S}}$ is the likelihood of the event being consistent with the signal hypothesis (a particular new physics scenario) and $L_{\mathrm{B}}$ is the likelihood of the event being consistent with the background hypothesis (Standard Model sources of lepton pair events with missing transverse momentum). The distributions depend on $\Delta m$ and on the parent particle mass, $m$.

In our previous publications we justified the inclusion of most of these variables and described how they were used [4]. In particular the $-q \cos \theta$ variable is not used in the selectron and chargino searches because the $t$-channel neutralino (selectron search) and sneutrino exchange (chargino search) contributions make this signal distribution model-dependent.

The new momentum asymmetry variable describes the correlation between the momenta of the two leptons and is thus complementary to the two scaled momentum variables which are implemented in the likelihood function as independent variables. It is correlated with the acolinearity variable, and so the information was included using a two-dimensional probability distribution of momentum asymmetry versus acolinearity. Sensitivity studies for the selectron and smuon searches justified this addition. However similar studies for the other channels (stau, chargino and charged Higgs), where momentum distributions are broader, showed no net gain in sensitivity and so for these channels the acolinearity distribution alone was used. Also for these channels, we removed the distinction between leptons being identified as electrons and muons and merged them into one category since we found that there was no significant difference in search sensitivity.

For the selectron search, we required that at least one lepton be identified as an electron and that no lepton in the event be compatible with being a muon (i.e. the other lepton was not identified as a muon and it was not muon-hadron ambiguous). Similarly for the smuon search we required at least one identified muon and required that no lepton be compatible with being

an electron. Some additional requirements were used to reject events from these searches if the second lepton was identified as a hadronically decaying tau or a "rest-of-event" candidate and if it had properties strongly incompatible with being an electron for the selectron search and with being a muon for the smuon search. These additional requirements cut the background by $21 \%$ and $14 \%$ while only reducing the signal efficiency by $2.1 \%$ and $0.8 \%$ for the selectron and smuon searches respectively.

For each search channel, reference histograms were constructed for each of the likelihood variables at each point in $m$ and $\Delta m$ for which signal Monte Carlo had been generated (each 
point corresponds to a table entry in tables 3-8). A smoothing algorithm [26] was applied to the histograms to reduce the effects of statistical fluctuations. The reference histograms were then used to construct $L_{\mathrm{R}}$ distributions. An interpolation procedure was developed which allowed us to interpolate among neighbouring signal Monte Carlo $(m, \Delta m, \sqrt{s})$ points to intermediate values of $(m, \Delta m$ and $\sqrt{s})$. Further details are given in [27].

$L_{\mathrm{R}}$ distributions for signal Monte Carlo, Standard Model Monte Carlo and data are shown in Figure 4 for the specific example of the analysis for staus with a mass of $80 \mathrm{GeV}$ and a stau-neutralino mass difference of $60 \mathrm{GeV}$. There is considerable variation in the shapes of these distributions with $m$ and $\Delta m$ but agreement between data and Standard Model Monte Carlo is generally good.

\subsection{Calculation of Cross-section Upper Limits}

For each search channel and for each set of kinematic parameters ( $m$ and $\Delta m$ ) we wish to test the consistency of the data with the sum of background and an additional signal contribution. The signal contribution depends on $\sigma_{\mathrm{s}}$, the cross-section multiplied by branching ratio to leptonic final states squared. We use an extended maximum likelihood technique as described in [4] to measure $\sigma_{\mathrm{s}}$ and, in the absence of a significant signal, to set $95 \%$ confidence level (CL) upper limits on $\sigma_{\mathrm{s}}$. We form a likelihood, $L\left(\sigma_{\mathrm{s}}\right)$, of the set of $L_{\mathrm{R}}$ values for the data being consistent with the expected $L_{\mathrm{R}}$ distribution for Standard Model plus a signal contribution of $\sigma_{\mathrm{s}}$. The upper limit on the cross-section multiplied by branching ratio squared at $95 \%$ confidence level, $\sigma_{95}$, is calculated as the value of $\sigma_{\mathrm{s}}$ below which $95 \%$ of the area under the likelihood function lies. Details of the likelihood function and method as well as cross-checks of the method are described in [4]. Data from different centre-of-mass energies are combined by weighting them using an assumed cross-section dependence on $\sqrt{s}$. For this paper the reference centre-of-mass energy for the weighting is $\sqrt{s}=208 \mathrm{GeV}$ instead of $\sqrt{s}=189 \mathrm{GeV}$.

We have also evaluated results based on the number of events passing an optimised cut on the $L_{\mathrm{R}}$ value. These results are used as a cross-check, as a direct comparison between data and background and as a simpler basis for combining this analysis with other experiments or other analyses.

\section{Constraints on New Physics}

We present limits on the pair production of charged scalar leptons, leptonically decaying charged Higgs bosons and charginos that decay to produce a charged lepton and invisible particles.

The $95 \%$ CL upper limit on new particle production at $\sqrt{s}=208 \mathrm{GeV}$ obtained by combining the data at different centre-of-mass energies is calculated at each kinematically allowed point on a $1 \mathrm{GeV}$ by $1 \mathrm{GeV}$ grid of $m$ and $\Delta m$, using the $L_{\mathrm{R}}$ distributions for signal and background, the $L_{\mathrm{R}}$ values of the data events, and the efficiency of the general selection at that point as input.

In addition to the Monte Carlo statistical error on the signal efficiency, we assess a 10\% relative systematic error on the estimated selection efficiency to take into account deficiencies in the Monte Carlo generators and the detector simulation (5\%), uncertainties in the interpolation procedure (5\%), effect of tau polarisation in stau decay [28] (5\%), fluctuations in the shape of 
the signal $L_{\mathrm{R}}$ distribution (2\%), uncertainties from lepton identification efficiency (2\%), detector occupancy $(1 \%)$, trigger efficiency $(<1 \%)$, and luminosity measurement $(0.5 \%)$.

At high values of $\Delta m$ the dominant background to the searches for new physics results from $\mathrm{W}^{+} \mathrm{W}^{-}$production. High statistics Monte Carlo samples for this process are available and these samples describe the OPAL data well [29]. In addition to the Monte Carlo statistical error, we assess a $5 \%$ relative systematic error on the estimated background to take into account uncertainties in the shapes of the $L_{\mathrm{R}}$ distributions and reference histograms, in the interpolation procedure, and deficiencies in the Monte Carlo detector simulation. At low values of $\Delta m$ the dominant background results from two-photon $\mathrm{e}^{+} \mathrm{e}^{-} \ell^{+} \ell^{-}$events. The background uncertainty at low $\Delta m$ is dominated by the limited Monte Carlo statistics; the uncertainty is typically $20-80 \%$ at low $\Delta m$.

We have examined distributions of the cut variables and background enriched samples in assessing these systematic errors. The systematic errors on the selection efficiency and the estimated background are considered as global errors applicable to each search and for all values of $m$ and $\Delta m$. In setting limits, the Monte Carlo statistical errors and the systematic uncertainties on the efficiency and the background expectation are taken into account using numerical convolution.

\subsection{Limits on $\sigma_{\mathrm{s}}$}

Limits on $\sigma_{\mathrm{s}}$, the production cross-section for new physics processes multiplied by the branching ratio squared, are presented in a manner intended to minimise the number of model assumptions. The 95\% CL upper limits at $\sqrt{s}=208 \mathrm{GeV}$ shown in Figures $5-10$ are obtained by combining the data at the various centre-of-mass energies using the assumption that the cross-section varies as $\beta^{3} / s$ for sleptons and charged Higgs and $\beta / s$ for charginos, where $\beta$ is the particle's velocity in units of $c$. The chosen functional forms are used for simplicity in presenting the data and represent an approximation, most importantly for processes in which t-channel exchange may be important, namely selectron pair and chargino pair production. In these cases the cross-section dependence on centre-of-mass energy is model dependent, depending on the mass of the exchanged particles and the couplings of the neutralinos and charginos. The selectron Monte Carlo events were generated with $\mu=-200 \mathrm{GeV}$ and $\tan \beta=1.5$ using Susygen. We have found by varying $\mu$ and $\tan \beta$, over the range $100<|\mu|<1000 \mathrm{GeV}$ and $1<\tan \beta<50$, that the above choice gives a conservative estimate of the selection efficiency for selectrons.

Upper limits at 95\% CL on the selectron pair cross-section at $\sqrt{s}=208 \mathrm{GeV}$ multiplied by the branching ratio squared for the decay $\tilde{\mathrm{e}}^{-} \rightarrow \mathrm{e}^{-} \tilde{\chi}_{1}^{0}$ are shown in Figure 5 as a function of selectron mass and lightest neutralino mass. These limits are applicable to $\tilde{\mathrm{e}}_{\mathrm{L}}^{+} \tilde{\mathrm{e}}_{\mathrm{L}}^{-}$and $\tilde{\mathrm{e}}_{\mathrm{R}}^{+} \tilde{\mathrm{e}}_{\mathrm{R}}^{-}$ production. The corresponding plots for the smuon and stau pair searches are shown in Figures 6 and 7 , respectively. Note that if the LSP is the effectively massless gravitino, $\tilde{G}$, then for prompt slepton decays to a lepton and a gravitino the experimental signature would be the same as that for $\tilde{\ell}^{-} \rightarrow \ell^{-} \tilde{\chi}_{1}^{0}$ with a massless neutralino. In this case the limits given in Figures $5-7$ for $m_{\tilde{\chi}_{1}^{0}}=0$ may be interpreted as limits on the decay $\tilde{\ell}^{-} \rightarrow \ell^{-} \tilde{G}$.

The upper limit at 95\% CL on the chargino pair production cross-section multiplied by the branching ratio squared for the decay $\tilde{\chi}_{1}^{ \pm} \rightarrow \ell^{ \pm} \tilde{\nu}_{\ell}$ (2-body decay) is shown in Figure 8 . The limit has been calculated for the case where the three sneutrino generations are mass degenerate. The 
upper limit at 95\% CL on the chargino pair production cross-section multiplied by the branching ratio squared for the decay $\tilde{\chi}_{1}^{ \pm} \rightarrow \mathrm{W}^{ \pm} \tilde{\chi}_{1}^{0} \rightarrow \ell^{ \pm} \nu \tilde{\chi}_{1}^{0}$ (3-body decay) is shown in Figure 9.

The upper limit at 95\% CL on the charged Higgs boson pair production cross-section multiplied by the branching ratio squared for the decay $\mathrm{H}^{ \pm} \rightarrow \tau^{ \pm} \nu_{\tau}$ is shown as a function of $m_{\mathrm{H}^{+}}$ as the solid histogram in Figure 10. The branching ratio for the decay $\mathrm{H}^{ \pm} \rightarrow \tau^{ \pm} \nu_{\tau}$ may be the dominant one for the charged Higgs masses explored with this data-set. The dashed line in Figure 10 shows the prediction from HZHA at $\sqrt{s}=208 \mathrm{GeV}$ for a $100 \%$ branching ratio for the decay $\mathrm{H}^{ \pm} \rightarrow \tau^{ \pm} \nu_{\tau}$. With this assumption we set a lower limit at $95 \% \mathrm{CL}$ on $m_{\mathrm{H}^{+}}$of $92.0 \mathrm{GeV}$.

\subsection{Expected Limits and Consistency with Expectation}

For each search, we provide a table showing quantitatively the signal efficiencies and the agreement of the data with the Standard Model background expectations for a number of values of $m$ and $\Delta m$. Tables 3, 4, 5,6,7 and 8 give the values of the following quantities in the searches for selectrons, smuons, staus, charginos with two-body decay, charginos with three-body decay and charged Higgs, respectively:

1. The signal efficiency for the general selection at $208 \mathrm{GeV}$ with statistical error (the efficiencies at lower energies differ by less than $3 \%$ ).

2. The $95 \%$ CL upper limit on the cross-section multiplied by the branching ratio squared at $208 \mathrm{GeV}$, obtained by combining the data from each centre-of-mass energy.

3. The expected 95\% CL upper limit on the cross-section multiplied by the branching ratio squared in the absence of signal, $\left\langle\sigma_{95}\right\rangle$. This is calculated using an ensemble of 1000 Monte Carlo experiments to simulate the data. In each Monte Carlo experiment, the total number of candidates is drawn from a Poisson distribution with mean equal to the number of events expected from the Standard Model. For each candidate, a value of $L_{\mathrm{R}}$ is assigned, chosen randomly according to the expected $L_{\mathrm{R}}$ distribution for Standard Model processes. The expected limit at a given point in $m$ and $\Delta m$ is the mean value of the limit for the ensemble of simulated experiments.

4. The confidence level for consistency with the Standard Model, calculated as the fraction of the simulated experiments for which the upper limit on the cross-section multiplied by the branching ratio squared is greater than or equal to the value calculated using the actual data. In the absence of signal, a CL of $50 \%$ is expected on average ${ }^{7}$.

Note that the number of candidate events with high values of relative likelihood varies greatly from search to search and with $m$ and $\Delta m$. For the selectron and smuon searches where particular di-lepton identities are required and the kinematics of the lepton from the slepton decay are measured directly (in contrast to events with tau leptons), relatively few events may be present at high relative likelihood. For searches where the signal di-lepton identities are not

${ }^{7}$ Values of $100 \%$ correspond to points where there are no candidate events with non-zero $L_{\mathrm{R}}$ in the OPAL data. In this case, all toy Monte Carlo experiments will have a value of $\sigma_{95}$ equal to or (if there are Monte Carlo candidates with non-zero $L_{\mathrm{R}}$ ) greater than the value for the data. 
that dissimilar to $\mathrm{W}^{+} \mathrm{W}^{-} \rightarrow \ell^{+} \nu \ell^{-} \bar{\nu}$ and a range of lepton momenta are expected, hundreds of events may potentially have high values of relative likelihood.

For some points in $m$ and $\Delta m$ in these tables, the confidence level for consistency with the Standard Model is relatively small (of order 1\%). The probability of getting a low confidence level for one or more points in $m$ and $\Delta m$ for one or more of the search channels depends on the degree of correlation among the different $(m, \Delta m)$ points and among the different channels. The correlation between adjacent points is strong when the momentum distributions for those points are similar. The momentum distributions vary slowly with both $m$ and $\Delta m$ when $\Delta m$ is high (hence the clustering of low confidence level values in Table 4), but vary considerably with $\Delta m$ when $\Delta m$ is low.

This effect was investigated by generating 1000 Monte Carlo experiments with the Standard Model Monte Carlo as described above. For each experiment and each point on the $(m, \Delta m)$ grid at which signal Monte Carlo has been generated, the CL was calculated. It was found that $56 \%$ of the experiments had at least one point with a CL of $0.9 \%$ or less; this is the lowest value observed in Tables 3 to 8 .

\subsection{Limits on Slepton Masses}

We can use our data to set limits on the masses of right-handed sleptons ${ }^{8}$ based on the expected right-handed slepton pair production cross-sections and branching ratios. The cross-sections were calculated using SUSYGEN at each centre-of-mass energy and take into account initial state radiation. In Figure 11 we show limits on right-handed smuons as a function of smuon mass and lightest neutralino mass for several assumed values of the branching ratio squared for $\tilde{\mu}_{R}^{ \pm} \rightarrow \mu^{ \pm} \tilde{\chi}_{1}^{0}$. The expected limit, calculated using Standard Model Monte Carlo only, is also shown for a branching ratio of $100 \%$. For a branching ratio $\tilde{\mu}_{R}^{ \pm} \rightarrow \mu^{ \pm} \tilde{\chi}_{1}^{0}$ of $100 \%$ and for a smuon-neutralino mass difference exceeding $4 \mathrm{GeV}$, right-handed smuons are excluded at $95 \%$ CL for masses below $94.0 \mathrm{GeV}$. The $95 \% \mathrm{CL}$ upper limit on the production of right-handed $\tilde{\tau}^{+} \tilde{\tau}^{-}$multiplied by the branching ratio squared for $\tilde{\tau}_{R}^{ \pm} \rightarrow \tau^{ \pm} \tilde{\chi}_{1}^{0}$ is shown in Figure 12 . The expected limit for a branching ratio of $100 \%$ is also shown. For a branching ratio $\tilde{\tau}_{R}^{ \pm} \rightarrow \tau^{ \pm} \tilde{\chi}_{1}^{0}$ of $100 \%$ and for a stau-neutralino mass difference exceeding $8 \mathrm{GeV}$, right-handed staus are excluded at $95 \% \mathrm{CL}$ for masses below $89.8 \mathrm{GeV}$. No mixing between $\tilde{\tau}_{L}$ and $\tilde{\tau}_{R}$ is assumed. However, the cross-section ratio $\sigma_{\tilde{\tau}_{1}^{+} \tilde{\tau}_{1}^{-}} / \sigma_{\tilde{\tau}_{R}^{+}} \tilde{\tau}_{R}^{-}$at $\sqrt{s} \approx 208 \mathrm{GeV}$ varies between 0.90 and 1.17 depending only on the mixing angle. Using this information, the limits shown in Figure 12 can be applied to any degree of stau mixing by multiplying the predicted cross-section for $\tilde{\tau}_{R}^{+} \tilde{\tau}_{R}^{-}$by the value of $\sigma_{\tilde{\tau}_{1}^{+} \tilde{\tau}_{1}^{-}} / \sigma_{\tilde{\tau}_{R}^{+} \tilde{\tau}_{R}^{-}}$corresponding to the mixing angle considered. The two broken lines in Figure 12 show the range of possible positions of the line defining the excluded region for a branching ratio $\tilde{\tau}_{1}^{ \pm} \rightarrow \tau^{ \pm} \tilde{\chi}_{1}^{0}$ of $100 \%$ for any degree of stau mixing.

For the case of a massless neutralino (or gravitino) and $100 \%$ branching ratio, right-handed smuons and staus are excluded at 95\% CL for masses below $94.3 \mathrm{GeV}$ and $89.8 \mathrm{GeV}$, respectively, and $\tilde{\tau}_{1}^{ \pm}$is excluded at $95 \%$ CL for masses below $88.7 \mathrm{GeV}$, for any degree of stau mixing.

An alternative approach is to set limits taking into account the predicted cross-section and branching ratio for specific choices of the parameters within the Minimal Supersymmetric

\footnotetext{
${ }^{8}$ The right-handed slepton is expected to be lighter than the left-handed slepton. The right-handed one tends (not generally valid for selectrons) to have a lower pair production cross-section, and so conventionally limits are given for this (usually) conservative case.
} 
Standard Model (MSSM) $)^{9}$. For $\mu<-100 \mathrm{GeV}$ and for two values of $\tan \beta$ (1.5 and 35), Figures 13, 14 and 15 show 95\% CL exclusion regions in the $\left(m_{\tilde{\ell}_{\mathrm{R}}^{ \pm}}, m_{\tilde{\chi}_{1}^{0}}\right)$ plane for righthanded selectrons, smuons and staus, respectively. For $\mu<-100 \mathrm{GeV}$ and $\tan \beta=1.5$, righthanded sleptons are excluded at 95\% CL as follows: selectrons with masses below $97.5 \mathrm{GeV}$ for $m_{\tilde{\mathrm{e}}^{-}}-m_{\tilde{\chi}_{1}^{0}}>11 \mathrm{GeV}$; smuons with masses below $91.0 \mathrm{GeV}$ for $m_{\tilde{\mu}^{-}}-m_{\tilde{\chi}_{1}^{0}}>3 \mathrm{GeV}$; and staus with masses below $85.2 \mathrm{GeV}$ for $m_{\tilde{\tau}^{-}}-m_{\tilde{\chi}_{1}^{0}}>6 \mathrm{GeV}$.

\subsection{Search for Unequal Mass Particle Production}

All the search results described above are for the pair production of new particles with equal mass. New physics signals with unequal mass, such as the production of $\tilde{\mathrm{e}}_{L} \tilde{\mathrm{e}}_{R}$ in which the two selectrons have different mass and each selectron decays to e $\tilde{\chi}_{1}^{0}$, can potentially be observed in the acoplanar di-lepton event sample.

We have examined the data for di-electron, di-muon and arbitrary di-lepton identity events consistent with unequal mass particle production. The search hypothesis is $\mathrm{e}^{+} \mathrm{e}^{-} \rightarrow \mathrm{XY}$, with subsequent decays, $\mathrm{X} \rightarrow \ell^{ \pm} \mathrm{N}$ and $\mathrm{Y} \rightarrow \ell^{\mp} \mathrm{N}$, where $\mathrm{X}$ and $\mathrm{Y}$ are two massive charged particles, and $\mathrm{N}$ is an invisible particle such as the LSP. The free parameters in the search are the masses of particles X, Y and N. The kinematics of producing two particles with different mass constrain the lepton momenta to be within well defined ranges depending on the new particle masses. The data have been examined by scanning the three particle masses in steps of $10 \mathrm{GeV}$ for the three different di-lepton identity hypotheses and requiring the measured lepton momenta to be within the ranges specified by kinematics.

The consistency of the data with the Standard Model expectations is then examined. The lowest probability mass hypothesis occurs for di-leptons of arbitrary flavour with particle mass $m_{\mathrm{X}}=130 \mathrm{GeV}, m_{\mathrm{Y}}=60 \mathrm{GeV}$ and $m_{\mathrm{N}}=20 \mathrm{GeV}$, where 242 events are observed with 197.3 expected from Standard Model sources (Poisson probability of $0.7 \%$ ). From an ensemble of 1000 Standard Model Monte Carlo experiments it is found that $12.5 \%$ of the experiments had at least one mass hypothesis with a Poisson probability of $0.7 \%$ or lower. In general, the agreement with expectation is good. In conclusion, we have explored this potential weakness of the standard equal mass pair production search and found no significant evidence for unequal mass particle production.

\section{Summary and Conclusions}

A selection of di-lepton events with significant missing transverse momentum is performed using a data sample with an integrated luminosity of $680 \mathrm{pb}^{-1}$ collected at $\mathrm{e}^{+} \mathrm{e}^{-}$centre-of-mass energies ranging from 183 to $208 \mathrm{GeV}$. The observed number of events, 1317, the dependence on centre-of-mass energy and the event properties are consistent with expectations from Standard Model processes, dominantly arising from $\mathrm{W}^{+} \mathrm{W}^{-}$production with both $\mathrm{W}$ bosons decaying leptonically.

\footnotetext{
${ }^{9}$ In particular regions of the MSSM parameter space, the branching ratio for $\tilde{\ell}^{ \pm} \rightarrow \ell^{ \pm} \tilde{\chi}_{1}^{0}$ can be essentially zero as a result of competing cascade decays and so it is not possible to provide general limits on sleptons within the MSSM on the basis of this search alone. The predicted cross-sections and branching ratios within the MSSM are obtained using SUSYGEN and are calculated with the gauge unification relation, $M_{1}=\frac{5}{3} \tan ^{2} \theta_{W} M_{2}$.
} 
Discrimination techniques are employed to search for the pair production of charged scalar leptons, leptonically decaying charged Higgs bosons and charginos that decay to produce a charged lepton and invisible particles. No evidence for new phenomena is apparent. Upper limits on the production cross-section multiplied by the branching ratio squared for each new physics process are presented in a manner intended to minimise the number of model assumptions.

Assuming a $100 \%$ branching ratio for the decay $\tilde{\ell}_{R}^{ \pm} \rightarrow \ell^{ \pm} \tilde{\chi}_{1}^{0}$, we exclude at $95 \%$ CL: righthanded smuons with masses below $94.0 \mathrm{GeV}$ for $m_{\tilde{\mu}^{-}}-m_{\tilde{\chi}_{1}^{0}}>4 \mathrm{GeV}$ and right-handed staus with masses below $89.8 \mathrm{GeV}$ for $m_{\tilde{\tau}^{-}}-m_{\tilde{\chi}_{1}^{0}}>8 \mathrm{GeV}$. Right-handed selectrons are excluded at $95 \% \mathrm{CL}$ for masses below $97.5 \mathrm{GeV}$ for $m_{\tilde{\mathrm{e}}^{-}}-m_{\tilde{\chi}_{1}^{0}}>11 \mathrm{GeV}$ within the framework of the MSSM assuming $\mu<-100 \mathrm{GeV}$ and $\tan \beta=1.5$. Charged Higgs bosons are excluded at $95 \%$ CL for masses below $92.0 \mathrm{GeV}$, assuming a $100 \%$ branching ratio for the decay $\mathrm{H}^{ \pm} \rightarrow \tau^{ \pm} \nu_{\tau}$.

\section{Acknowledgements}

We particularly wish to thank the SL Division for the efficient operation of the LEP accelerator at all energies and for their close cooperation with our experimental group. In addition to the support staff at our own institutions we are pleased to acknowledge the

Department of Energy, USA, National Science Foundation, USA, Particle Physics and Astronomy Research Council, UK, Natural Sciences and Engineering Research Council, Canada, Israel Science Foundation, administered by the Israel Academy of Science and Humanities, Benoziyo Center for High Energy Physics,

Japanese Ministry of Education, Culture, Sports, Science and Technology (MEXT) and a grant under the MEXT International Science Research Program, Japanese Society for the Promotion of Science (JSPS), German Israeli Bi-national Science Foundation (GIF), Bundesministerium für Bildung und Forschung, Germany, National Research Council of Canada, Hungarian Foundation for Scientific Research, OTKA T-038240, and T-042864, The NWO/NATO Fund for Scientific Research, the Netherlands.

\section{References}

[1] H.P. Nilles, Phys. Rep. 110 (1984) 1;

H.E. Haber and G.L. Kane, Phys. Rep. 117 (1985).

[2] OPAL Collab., K. Ackerstaff et al., Eur. Phys. J. C4 (1998) 47.

[3] OPAL Collab., G. Abbiendi et al., Eur. Phys. J. C12 (2000) 551.

[4] OPAL Collab., G. Abbiendi et al., Eur. Phys. J. C14 (2000) 51.

[5] ALEPH Collab., A. Heister et al., Phys. Lett. B526 (2002) 206. 
[6] L3 Collab., M. Acciarri et al., Phys. Lett. B471 (1999) 280;

DELPHI Collab., P. Abreu et al., Eur. Phys. J. C19 (2001) 29.

[7] OPAL Collab., K. Ahmet et al., Nucl. Instr. Meth. A305 (1991) 275;

S. Anderson, Nucl. Instr. Meth. A403 (1998) 326.

[8] G. Aguillion et al., Nucl. Instr. Meth. A417 (1998) 266.

[9] S. Jadach et al., Comp. Phys. Comm. 119 (1999) 272.

[10] J. Fujimoto et al., Comp. Phys. Comm. 100 (1997) 128;

J. Fujimoto et al., in Physics at LEP2, edited by G. Altarelli, T. Sjöstrand and F. Zwirner, CERN 96-01, Vol. 2 (1996) p. 30.

[11] F.A. Berends, P.H. Daverveldt and R. Kleiss, Comp. Phys. Comm. 40 (1986) 271.

[12] J.A.M. Vermaseren, Nucl. Phys. B229 (1983) 347.

[13] Phojet 1.05 generator: E. Budinov et al., in Physics at LEP2, edited by G. Altarelli, T. Sjöstrand and F. Zwirner, CERN 96-01, Vol. 2 (1996) p. 216;

R. Engel and J. Ranft, Phys. Rev. D54 (1996) 4244.

[14] G. Marchesini et al., Comp. Phys. Comm. 67 (1992) 465.

[15] S. Jadach, W. Płaczek and B.F.L. Ward, Phys. Lett. 390 (1997) 298.

[16] D. Karlen, Nucl. Phys. B289 (1987) 23.

[17] S. Jadach, B.F. Ward and Z. Wąs, Phys. Rev. D63 (2001) 113009.

[18] G. Montagna, M. Moretti, O. Nicrosini and F. Piccinini, Nucl. Phys. B541 (1999) 31.

[19] S. Katsanevas and S. Melachroinos, in Physics at LEP2, edited by G. Altarelli, T. Sjöstrand and F. Zwirner, CERN 96-01, Vol. 2 (1996) p. 216. S. Katsanevas and P. Morawitz, Comp. Phys. Comm. 112 (1998) 227.

[20] C. Dionisi et al., in "Physics at LEP2", edited by G. Altarelli, T. Sjöstrand and F. Zwirner, CERN 96-01, Vol. 2 (1996) p. 337.

[21] P. Janot, in "Physics at LEP2", edited by G. Altarelli, T. Sjöstrand and F. Zwirner, CERN 96-01, Vol. 2 (1996) p. 309.

[22] M. Arignon et al., Nucl. Instr. Meth. A313 (1992) 103.

[23] J. Allison et al., Nucl. Instr. Meth. A317 (1992) 47.

[24] OPAL Collab., R. Akers et al., Z. Phys. C63 (1994) 197.

[25] OPAL Collab., G. Abbiendi et al., Eur. Phys. J. C21 (2001) 1.

[26] J. Allison, Comp. Phys. Comm. 77 (1993) 377.

[27] T.E. Marchant, "Search for New Physics Using Acoplanar Lepton Pair Events in $\mathrm{e}^{+} \mathrm{e}^{-}$ Collisions at $\sqrt{s}=183-208 \mathrm{GeV} "$, University of Manchester Ph.D. Thesis, January 2002. 
[28] M.M. Nojiri, Phys. Rev. D51 (1995) 6281.

[29] OPAL Collab., G. Abbiendi et al., Eur. Phys. J. C8 (1999) 191. 


\begin{tabular}{|c|c|c|c|c|c|c|c|c|c|c|c|c|c|}
\hline \multirow{2}{*}{$\begin{array}{l}\Delta m \\
(\mathrm{GeV})\end{array}$} & \multicolumn{13}{|c|}{$m_{\tilde{e}^{-}}(\mathrm{GeV})$} \\
\hline & 45 & 50 & 55 & 60 & 65 & 70 & 75 & 80 & 85 & 90 & 94 & 99 & 103 \\
\hline \multicolumn{14}{|c|}{ "General Selection Efficiency ( \% ) } \\
\hline 2 & $5.0 \pm 0.7$ & $3.8 \pm 0.6$ & $4.5 \pm 0.7$ & $2.2 \pm 0.5$ & $2.0 \pm 0.4$ & $1.6 \pm 0.4$ & $1.2 \pm 0.3$ & $0.8 \pm 0.3$ & $0.6 \pm 0.2$ & $0.5 \pm 0.2$ & $0.5 \pm 0.2$ & $0.1 \pm 0.1$ & $0.0 \pm 0.0$ \\
\hline 2.5 & $5.0 \pm 0.7$ & $3.8 \pm 0.6$ & $4.5 \pm 0.7$ & $2.2 \pm 0.5$ & $2.0 \pm 0.4$ & $1.6 \pm 0.4$ & $1.2 \pm 0.3$ & $0.8 \pm 0.3$ & $0.6 \pm 0.2$ & $0.5 \pm 0.2$ & $0.5 \pm 0.2$ & $0.1 \pm 0.1$ & $0.0 \pm 0.0$ \\
\hline 5 & $40.7 \pm 1.6$ & $42.9 \pm 1.6$ & $41.9 \pm 1.6$ & $44.5 \pm 1.6$ & $45.0 \pm 1.6$ & $43.5 \pm 1.6$ & $43.1 \pm 1.6$ & $39.9 \pm 1.5$ & $41.6 \pm 1.6$ & $44.1 \pm 1.6$ & $43.1 \pm 1.6$ & $42.3 \pm 1.6$ & $42.4 \pm 1.6$ \\
\hline 10 & $60.9 \pm 1.5$ & $64.8 \pm 1.5$ & $65.4 \pm 1.5$ & $66.4 \pm 1.5$ & $67.7 \pm 1.5$ & $67.2 \pm 1.5$ & $65.9 \pm 1.5$ & $67.8 \pm 1.5$ & $66.0 \pm 1.5$ & $67.4 \pm 1.5$ & $67.4 \pm 1.5$ & $66.6 \pm 1.5$ & $67.8 \pm 1.5$ \\
\hline 20 & $74.8 \pm 1.4$ & $76.3 \pm 1.3$ & $77.4 \pm 1.3$ & $79.1 \pm 1.3$ & $81.2 \pm 1.2$ & $82.4 \pm 1.2$ & $82.5 \pm 1.2$ & $81.1 \pm 1.2$ & $81.4 \pm 1.2$ & $81.3 \pm 1.2$ & $82.1 \pm 1.2$ & $83.3 \pm 1.2$ & $80.2 \pm 1.3$ \\
\hline$m / 2$ & $75.0 \pm 1.4$ & $78.6 \pm 1.3$ & $79.4 \pm 1.3$ & $83.2 \pm 1.2$ & $85.9 \pm 1.1$ & $84.4 \pm 1.1$ & $85.5 \pm 1.1$ & $87.8 \pm 1.0$ & $88.2 \pm 1.0$ & $87.8 \pm 1.0$ & $88.5 \pm 1.0$ & $88.5 \pm 1.0$ & $89.4 \pm 1.0$ \\
\hline$m-20$ & $74.9 \pm 1.4$ & $81.7 \pm 1.2$ & $82.1 \pm 1.2$ & $83.6 \pm 1.2$ & $84.2 \pm 1.2$ & $84.9 \pm 1.1$ & $88.4 \pm 1.0$ & $86.0 \pm 1.1$ & $88.1 \pm 1.0$ & $89.9 \pm 1.0$ & $90.9 \pm 0.9$ & $90.2 \pm 0.9$ & $90.8 \pm 0.9$ \\
\hline$m-10$ & $74.6 \pm 1.4$ & $79.3 \pm 1.3$ & $79.8 \pm 1.3$ & $82.0 \pm 1.2$ & $85.2 \pm 1.1$ & $85.2 \pm 1.1$ & $89.1 \pm 1.0$ & $89.2 \pm 1.0$ & $88.7 \pm 1.0$ & $90.7 \pm 0.9$ & $90.1 \pm 0.9$ & $90.3 \pm 0.9$ & $90.5 \pm 0.9$ \\
\hline$m$ & $69.5 \pm 1.5$ & $77.3 \pm 1.3$ & $80.8 \pm 1.2$ & $83.1 \pm 1.2$ & $85.0 \pm 1.1$ & $88.2 \pm 1.0$ & $87.3 \pm 1.1$ & $89.2 \pm 1.0$ & $89.5 \pm 1.0$ & $88.5 \pm 1.0$ & $91.1 \pm 0.9$ & $90.4 \pm 0.9$ & $91.1 \pm 0.9$ \\
\hline
\end{tabular}

95\% CL upper limit on cross-section times $B R^{2}\left(\tilde{\mathrm{e}}^{-} \rightarrow \mathrm{e} \tilde{\chi}^{0}\right)(\mathrm{fb})$

\begin{tabular}{|c|c|c|c|c|c|c|c|c|c|c|c|c|c|}
\hline 2 & 82.1 & 86.6 & 99.4 & 139 & 113 & 160 & 294 & 412 & 1230 & 2530 & 5020 & - & $\overline{-}$ \\
\hline 2.5 & 34.0 & 33.7 & 36.3 & 39.6 & 43.5 & 35.4 & 43.7 & 58.6 & 85.9 & 136 & 256 & 975 & - \\
\hline 5 & 13.8 & 11.6 & 12.1 & 12.3 & 9.6 & 15.0 & 10.3 & 11.9 & 12.7 & 21.4 & 23.2 & 36.9 & 270 \\
\hline 10 & 14.0 & 17.2 & 12.9 & 9.7 & 10.2 & 10.3 & 10.0 & 10.2 & 10.6 & 11.1 & 14.9 & 23.9 & 174 \\
\hline 20 & 26.9 & 23.4 & 21.8 & 19.1 & 16.6 & 21.0 & 21.5 & 16.5 & 13.2 & 13.0 & 14.1 & 22.6 & 162 \\
\hline$m / 2$ & 34.4 & 35.0 & 37.2 & 34.6 & 34.2 & 27.9 & 25.7 & 31.0 & 31.2 & 37.5 & 40.3 & 75.6 & 282 \\
\hline$m-20$ & 39.6 & 43.1 & 49.7 & 45.7 & 47.6 & 53.7 & 60.8 & 47.7 & 35.2 & 30.2 & 34.3 & 60.1 & 243 \\
\hline$m-10$ & 48.3 & 56.3 & 56.1 & 56.5 & 49.4 & 48.1 & 61.7 & 54.8 & 42.2 & 33.9 & 40.3 & 52.9 & 228 \\
\hline$m$ & 56.0 & 59.3 & 61.3 & 52.0 & 51.2 & 46.7 & 53.9 & 62.9 & 39.7 & 36.5 & 37.9 & 54.8 & 229 \\
\hline \multicolumn{14}{|c|}{ expected upper limit on cross-section times $B R^{2}\left(\tilde{\mathrm{e}}^{-} \rightarrow \mathrm{e} \tilde{\chi}_{1}^{0}\right)(\mathrm{fb})$} \\
\hline 2 & 78.1 & 83.2 & 98.5 & 122 & 143 & 192 & 342 & 474 & 1360 & 2730 & 5280 & - & - \\
\hline 2.5 & 34.4 & 38.1 & 39.8 & 42.0 & 43.4 & 46.8 & 56.0 & 72.8 & 98.9 & 150 & 275 & 1010 & - \\
\hline 5 & 15.6 & 15.4 & 15.5 & 15.4 & 14.4 & 15.0 & 15.6 & 15.9 & 16.4 & 20.0 & 27.0 & 41.1 & 274 \\
\hline 10 & 18.0 & 15.6 & 14.4 & 13.4 & 12.7 & 12.6 & 12.1 & 12.4 & 13.2 & 16.0 & 19.9 & 29.4 & 188 \\
\hline 20 & 29.9 & 26.7 & 24.1 & 22.1 & 21.3 & 18.6 & 17.6 & 17.2 & 17.7 & 19.3 & 21.8 & 27.3 & 171 \\
\hline$m / 2$ & 34.0 & 32.9 & 32.2 & 32.8 & 31.9 & 32.9 & 33.4 & 35.3 & 38.9 & 42.1 & 43.7 & 45.9 & 191 \\
\hline$m-20$ & 36.0 & 37.1 & 37.8 & 39.1 & 40.2 & 42.6 & 44.8 & 48.4 & 48.3 & 49.1 & 50.8 & 54.9 & 219 \\
\hline$m-10$ & 38.0 & 38.3 & 37.2 & 38.5 & 40.3 & 44.6 & 46.4 & 49.5 & 48.8 & 47.7 & 51.1 & 55.0 & 218 \\
\hline$m$ & 38.9 & 37.5 & 37.9 & 38.9 & 40.8 & 43.0 & 47.5 & 49.7 & 47.7 & 48.8 & 49.7 & 53.9 & 216 \\
\hline \multicolumn{14}{|c|}{ CL for consistency with SM (\%) } \\
\hline 2 & 33.4 & 34.1 & 39.3 & 24.0 & 100.0 & 100.0 & 100.0 & 100.0 & 100.0 & 100.0 & 100.0 & - & - \\
\hline 2.5 & 42.6 & 57.2 & 53.4 & 46.4 & 42.8 & 63.1 & 100.0 & 100.0 & 100.0 & 100.0 & 100.0 & 100.0 & - \\
\hline 5 & 57.7 & 73.5 & 72.8 & 68.0 & 85.2 & 44.5 & 87.2 & 73.9 & 71.2 & 35.4 & 100.0 & 100.0 & 100.0 \\
\hline 10 & 72.9 & 32.2 & 56.0 & 81.0 & 67.8 & 66.8 & 63.3 & 69.0 & 68.6 & 88.9 & 100.0 & 66.3 & 100.0 \\
\hline 20 & 54.3 & 59.4 & 54.8 & 58.4 & 69.9 & 30.6 & 22.8 & 47.6 & 76.0 & 87.4 & 95.3 & 64.2 & 40.1 \\
\hline$m / 2$ & 42.2 & 36.2 & 26.4 & 37.2 & 36.1 & 63.4 & 74.5 & 58.4 & 67.4 & 55.1 & 52.5 & 5.3 & 7.2 \\
\hline$m-20$ & 32.3 & 27.5 & 17.5 & 28.1 & 24.9 & 19.6 & 14.9 & 45.6 & 79.5 & 91.4 & 85.1 & 32.3 & 27.1 \\
\hline$m-10$ & 18.8 & 8.4 & 7.3 & 9.4 & 20.9 & 34.5 & 14.2 & 32.4 & 62.0 & 79.6 & 70.4 & 45.9 & 33.6 \\
\hline$m$ & 9.9 & 5.3 & 4.4 & 16.3 & 21.5 & 33.7 & 30.5 & 18.8 & 65.6 & 75.5 & 73.6 & 42.0 & 34.2 \\
\hline
\end{tabular}




\begin{tabular}{|c|c|c|c|c|c|c|c|c|c|c|c|c|c|}
\hline \multirow{2}{*}{$\begin{array}{l}\Delta m \\
(\mathrm{GeV})\end{array}$} & \multicolumn{13}{|c|}{$m_{\tilde{\mu}^{-}}(\mathrm{GeV})$} \\
\hline & 45 & 50 & 55 & 60 & 65 & 70 & 75 & 80 & 85 & 90 & 94 & 99 & 103 \\
\hline \multicolumn{14}{|c|}{ General Selection Efficiency ( \% ) } \\
\hline 2 & $8.9 \pm 0.9$ & $8.5 \pm 0.9$ & $7.0 \pm 0.8$ & $4.3 \pm 0.6$ & $5.1 \pm 0.7$ & $2.7 \pm 0.5$ & $2.0 \pm 0.4$ & $0.5 \pm 0.2$ & $0.6 \pm 0.2$ & $0.2 \pm 0.1$ & $\overline{0.3 \pm 0.2}$ & $0.1 \pm 0.1$ & $0.0 \pm 0.0$ \\
\hline 2.5 & $8.9 \pm 0.9$ & $8.5 \pm 0.9$ & $7.0 \pm 0.8$ & $4.3 \pm 0.6$ & $5.1 \pm 0.7$ & $2.7 \pm 0.5$ & $2.0 \pm 0.4$ & $0.5 \pm 0.2$ & $0.6 \pm 0.2$ & $0.2 \pm 0.1$ & $0.3 \pm 0.2$ & $0.1 \pm 0.1$ & $0.0 \pm 0.0$ \\
\hline 5 & $53.1 \pm 1.6$ & $53.3 \pm 1.6$ & $50.7 \pm 1.6$ & $52.0 \pm 1.6$ & $51.3 \pm 1.6$ & $50.6 \pm 1.6$ & $47.0 \pm 1.6$ & $48.2 \pm 1.6$ & $48.4 \pm 1.6$ & $48.9 \pm 1.6$ & $47.4 \pm 1.6$ & $47.4 \pm 1.6$ & $46.4 \pm 1.6$ \\
\hline 10 & $71.1 \pm 1.4$ & $73.4 \pm 1.4$ & $70.6 \pm 1.4$ & $71.8 \pm 1.4$ & $70.1 \pm 1.4$ & $69.6 \pm 1.5$ & $70.3 \pm 1.4$ & $65.9 \pm 1.5$ & $73.3 \pm 1.4$ & $70.9 \pm 1.4$ & $69.9 \pm 1.5$ & $70.7 \pm 1.4$ & $70.0 \pm 1.4$ \\
\hline 20 & $82.6 \pm 1.2$ & $82.7 \pm 1.2$ & $84.4 \pm 1.1$ & $83.8 \pm 1.2$ & $84.9 \pm 1.1$ & $83.4 \pm 1.2$ & $85.7 \pm 1.1$ & $82.1 \pm 1.2$ & $84.7 \pm 1.1$ & $83.2 \pm 1.2$ & $83.3 \pm 1.2$ & $83.4 \pm 1.2$ & $83.5 \pm 1.2$ \\
\hline$m / 2$ & $84.0 \pm 1.2$ & $85.8 \pm 1.1$ & $86.5 \pm 1.1$ & $87.8 \pm 1.0$ & $90.3 \pm 0.9$ & $88.2 \pm 1.0$ & $89.2 \pm 1.0$ & $91.5 \pm 0.9$ & $92.1 \pm 0.9$ & $91.3 \pm 0.9$ & $91.3 \pm 0.9$ & $92.7 \pm 0.8$ & $92.5 \pm 0.8$ \\
\hline$m-20$ & $83.4 \pm 1.2$ & $87.1 \pm 1.1$ & $89.4 \pm 1.0$ & $89.6 \pm 1.0$ & $90.0 \pm 0.9$ & $91.3 \pm 0.9$ & $92.6 \pm 0.8$ & $93.4 \pm 0.8$ & $92.4 \pm 0.8$ & $93.5 \pm 0.8$ & $93.3 \pm 0.8$ & $92.9 \pm 0.8$ & $93.3 \pm 0.8$ \\
\hline$m-10$ & $88.6 \pm 1.0$ & $87.9 \pm 1.0$ & $89.2 \pm 1.0$ & $90.0 \pm 0.9$ & $91.8 \pm 0.9$ & $91.3 \pm 0.9$ & $93.0 \pm 0.8$ & $90.1 \pm 0.9$ & $93.2 \pm 0.8$ & $93.3 \pm 0.8$ & $93.5 \pm 0.8$ & $94.2 \pm 0.7$ & $94.6 \pm 0.7$ \\
\hline$m$ & $88.6 \pm 1.0$ & $88.8 \pm 1.0$ & $88.1 \pm 1.0$ & $91.3 \pm 0.9$ & $92.1 \pm 0.9$ & $93.3 \pm 0.8$ & $91.6 \pm 0.9$ & $93.5 \pm 0.8$ & $93.6 \pm 0.8$ & $92.8 \pm 0.8$ & $92.1 \pm 0.9$ & $93.5 \pm 0.8$ & $94.5 \pm 0.7$ \\
\hline
\end{tabular}

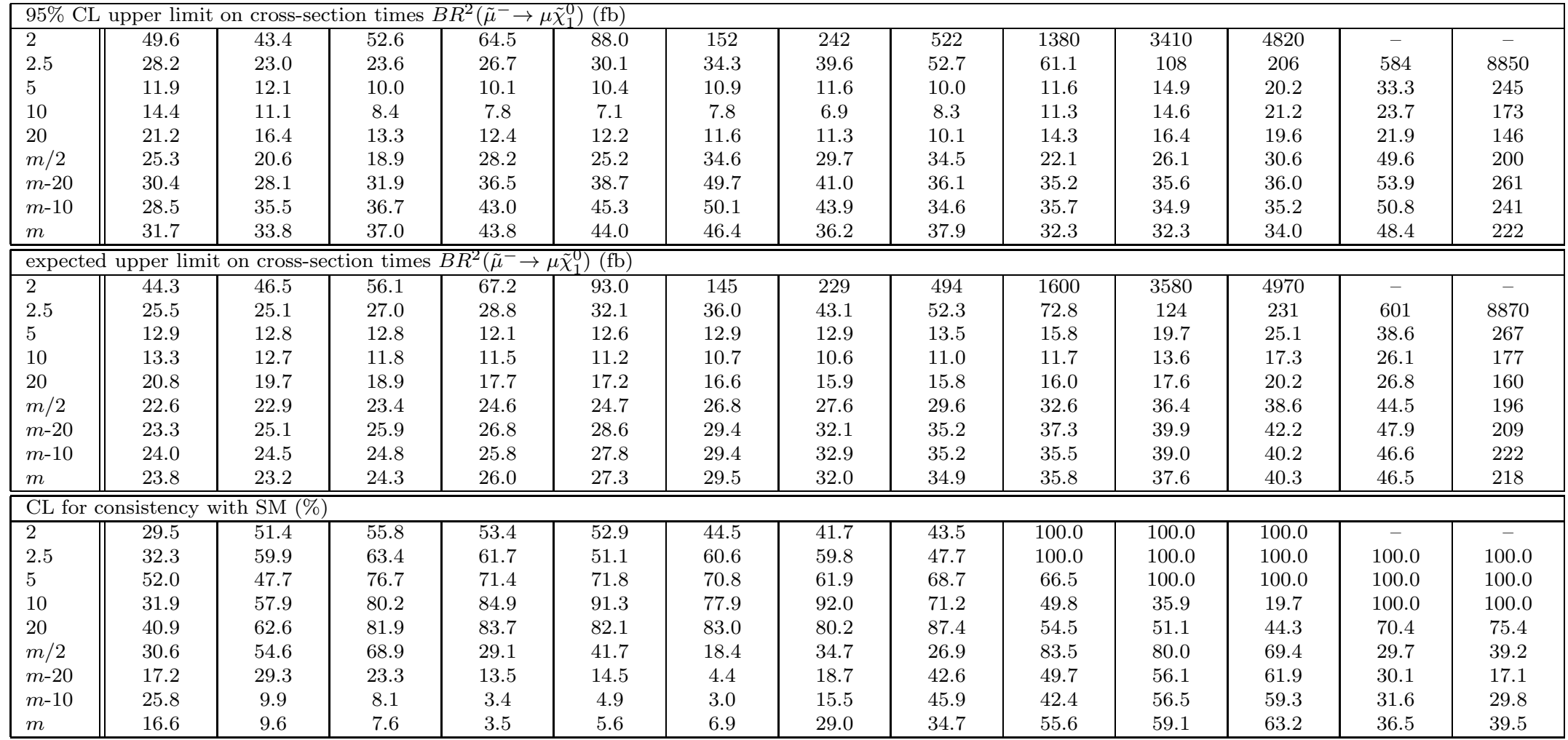




\begin{tabular}{|c|c|c|c|c|c|c|c|c|c|c|c|c|c|}
\hline \multirow{2}{*}{$\begin{array}{l}\Delta m \\
(\mathrm{GeV})\end{array}$} & \multicolumn{13}{|c|}{$m_{\tilde{\tau}^{-}}(\mathrm{GeV})$} \\
\hline & 45 & 50 & 55 & 60 & 65 & 70 & 75 & 80 & 85 & 90 & 94 & 99 & 103 \\
\hline \multicolumn{14}{|c|}{ General Selection Efficiency ( \% ) } \\
\hline 2 & $0.1 \pm 0.0$ & $0.0 \pm 0.0$ & $0.0 \pm 0.0$ & $0.0 \pm 0.0$ & $0.0 \pm 0.0$ & $0.0 \pm 0.0$ & $0.0 \pm 0.0$ & $0.0 \pm 0.0$ & $0.0 \pm 0.0$ & $0.0 \pm 0.0$ & $0.0 \pm 0.0$ & $0.0 \pm 0.0$ & $0.0 \pm 0.0$ \\
\hline 2.5 & $0.1 \pm 0.0$ & $0.0 \pm 0.0$ & $0.0 \pm 0.0$ & $0.0 \pm 0.0$ & $0.0 \pm 0.0$ & $0.0 \pm 0.0$ & $0.0 \pm 0.0$ & $0.0 \pm 0.0$ & $0.0 \pm 0.0$ & $0.0 \pm 0.0$ & $0.0 \pm 0.0$ & $0.0 \pm 0.0$ & $0.0 \pm 0.0$ \\
\hline 5 & $11.0 \pm 0.4$ & $10.5 \pm 0.4$ & $9.4 \pm 0.4$ & $8.5 \pm 0.4$ & $7.6 \pm 0.4$ & $7.6 \pm 0.4$ & $6.7 \pm 0.4$ & $6.4 \pm 0.3$ & $5.5 \pm 0.3$ & $4.5 \pm 0.3$ & $4.3 \pm 0.3$ & $4.0 \pm 0.3$ & $3.9 \pm 0.3$ \\
\hline 10 & $33.4 \pm 0.7$ & $33.8 \pm 0.7$ & $34.8 \pm 0.7$ & $32.5 \pm 0.7$ & $32.0 \pm 0.7$ & $31.8 \pm 0.7$ & $30.2 \pm 0.6$ & $30.9 \pm 0.7$ & $28.8 \pm 0.6$ & $26.6 \pm 0.6$ & $26.6 \pm 0.6$ & $25.0 \pm 0.6$ & $25.1 \pm 0.6$ \\
\hline 20 & $54.4 \pm 0.7$ & $56.0 \pm 0.7$ & $55.3 \pm 0.7$ & $56.4 \pm 0.7$ & $56.1 \pm 0.7$ & $54.8 \pm 0.7$ & $57.7 \pm 0.7$ & $55.5 \pm 0.7$ & $55.4 \pm 0.7$ & $54.8 \pm 0.7$ & $54.0 \pm 0.7$ & $55.0 \pm 0.7$ & $54.8 \pm 0.7$ \\
\hline$m / 2$ & $57.0 \pm 0.7$ & $60.0 \pm 0.7$ & $62.7 \pm 0.7$ & $65.5 \pm 0.7$ & $67.3 \pm 0.7$ & $69.4 \pm 0.7$ & $68.9 \pm 0.7$ & $70.3 \pm 0.6$ & $72.1 \pm 0.6$ & $74.0 \pm 0.6$ & $74.0 \pm 0.6$ & $74.6 \pm 0.6$ & $75.5 \pm 0.6$ \\
\hline$m-20$ & $59.4 \pm 0.7$ & $62.7 \pm 0.7$ & $66.5 \pm 0.7$ & $68.9 \pm 0.7$ & $71.1 \pm 0.6$ & $74.1 \pm 0.6$ & $75.7 \pm 0.6$ & $76.9 \pm 0.6$ & $76.4 \pm 0.6$ & $78.1 \pm 0.6$ & $79.3 \pm 0.6$ & $79.7 \pm 0.6$ & $79.5 \pm 0.6$ \\
\hline$m-10$ & $63.7 \pm 0.7$ & $67.1 \pm 0.7$ & $68.4 \pm 0.7$ & $72.5 \pm 0.6$ & $73.4 \pm 0.6$ & $74.8 \pm 0.6$ & $76.4 \pm 0.6$ & $76.9 \pm 0.6$ & $77.8 \pm 0.6$ & $77.5 \pm 0.6$ & $78.1 \pm 0.6$ & $79.4 \pm 0.6$ & $80.2 \pm 0.6$ \\
\hline$m$ & $66.5 \pm 0.7$ & $68.8 \pm 0.7$ & $70.1 \pm 0.6$ & $71.9 \pm 0.6$ & $75.1 \pm 0.6$ & $75.3 \pm 0.6$ & $76.9 \pm 0.6$ & $76.6 \pm 0.6$ & $78.9 \pm 0.6$ & $78.6 \pm 0.6$ & $79.9 \pm 0.6$ & $80.2 \pm 0.6$ & $80.2 \pm 0.6$ \\
\hline
\end{tabular}

95\% CL upper limit on cross-section times $B R^{2}\left(\tilde{\tau}^{-} \rightarrow \tau \tilde{\chi}_{1}^{0}\right)(\mathrm{fb})$

\begin{tabular}{|c|c|c|c|c|c|c|c|c|c|c|c|c|c|}
\hline 2 & 5120 & 6260 & - & - & - & - & - & - & - & - & - & - & - \\
\hline 2.5 & 2130 & 2750 & 2700 & 3020 & 4410 & 5060 & 5710 & 8190 & - & - & - & - & - \\
\hline 5 & 78.5 & 69.1 & 85.8 & 100 & 106 & 107 & 128 & 148 & 199 & 245 & 363 & 403 & 2960 \\
\hline 10 & 52.6 & 49.5 & 44.2 & 39.2 & 33.7 & 35.2 & 35.2 & 34.9 & 39.6 & 48.0 & 56.0 & 81.4 & 474 \\
\hline 20 & 50.3 & 42.5 & 42.8 & 40.3 & 40.8 & 36.9 & 36.7 & 39.0 & 37.2 & 34.8 & 37.5 & 42.3 & 235 \\
\hline$m / 2$ & 51.4 & 51.6 & 46.6 & 45.7 & 42.0 & 44.3 & 41.9 & 42.8 & 44.7 & 48.6 & 53.3 & 61.4 & 256 \\
\hline$m-20$ & 56.0 & 53.8 & 56.3 & 48.8 & 48.0 & 47.6 & 45.7 & 47.9 & 54.2 & 57.7 & 61.2 & 78.7 & 328 \\
\hline$m-10$ & 63.6 & 62.8 & 55.7 & 49.7 & 46.1 & 48.3 & 49.1 & 48.5 & 52.1 & 58.7 & 70.0 & 91.8 & 356 \\
\hline$m$ & 67.4 & 64.4 & 53.7 & 49.8 & 53.7 & 52.1 & 51.3 & 53.7 & 57.1 & 65.8 & 77.3 & 92.4 & 345 \\
\hline \multicolumn{14}{|c|}{ expected upper limit on cross-section times $B R^{2}\left(\tilde{\tau}^{-} \rightarrow \tau \tilde{\chi}_{1}^{0}\right)(\mathrm{fb})$} \\
\hline 2 & 5900 & 6190 & - & - & - & - & - & - & - & - & - & - & - \\
\hline 2.5 & 1690 & 2440 & 2460 & 3330 & 4590 & 6350 & 6460 & 10100 & - & - & - & - & - \\
\hline 5 & 86.6 & 87.9 & 94.9 & 105 & 113 & 120 & 141 & 159 & 193 & 265 & 351 & 586 & 3570 \\
\hline 10 & 44.0 & 42.0 & 41.0 & 42.6 & 43.0 & 45.0 & 49.0 & 50.3 & 56.3 & 68.0 & 78.0 & 114 & 646 \\
\hline 20 & 45.5 & 45.0 & 43.5 & 43.3 & 42.3 & 42.9 & 43.4 & 43.9 & 47.0 & 55.1 & 62.2 & 79.1 & 405 \\
\hline$m / 2$ & 47.0 & 48.5 & 48.0 & 50.0 & 49.3 & 50.7 & 51.9 & 55.2 & 61.0 & 71.4 & 82.2 & 110 & 505 \\
\hline$m-20$ & 49.4 & 49.9 & 51.3 & 52.1 & 53.2 & 55.3 & 58.5 & 60.6 & 66.0 & 79.0 & 87.9 & 118 & 538 \\
\hline$m-10$ & 52.8 & 52.5 & 53.1 & 53.8 & 56.0 & 56.9 & 59.4 & 61.5 & 68.6 & 79.2 & 92.1 & 119 & 535 \\
\hline$m$ & 53.6 & 52.8 & 53.2 & 55.3 & 55.8 & 58.1 & 61.2 & 62.9 & 69.0 & 78.7 & 91.1 & 118 & 535 \\
\hline \multicolumn{14}{|c|}{ CL for consistency with SM (\%) } \\
\hline 2 & 60.4 & 53.2 & - & - & - & - & - & - & - & - & - & - & - \\
\hline 2.5 & 17.6 & 28.4 & 31.7 & 54.2 & 49.2 & 80.4 & 65.9 & 61.2 & - & - & - & - & - \\
\hline 5 & 54.9 & 70.3 & 56.8 & 48.7 & 48.4 & 55.5 & 53.8 & 52.2 & 39.3 & 53.0 & 37.2 & 100.0 & 100.0 \\
\hline 10 & 23.3 & 26.2 & 34.0 & 51.8 & 72.7 & 71.3 & 80.0 & 83.2 & 81.4 & 80.4 & 79.0 & 84.6 & 77.3 \\
\hline 20 & 31.5 & 50.4 & 43.8 & 51.7 & 46.5 & 61.1 & 62.7 & 60.3 & 69.0 & 88.9 & 92.6 & 97.4 & 98.8 \\
\hline$m / 2$ & 33.7 & 35.7 & 46.3 & 55.4 & 61.7 & 59.5 & 69.2 & 73.5 & 78.8 & 84.1 & 88.6 & 95.5 & 99.5 \\
\hline$m-20$ & 30.4 & 35.7 & 34.5 & 51.8 & 54.6 & 60.4 & 72.9 & 70.9 & 66.1 & 78.6 & 82.2 & 86.3 & 92.5 \\
\hline$m-10$ & 23.7 & 24.2 & 38.2 & 54.3 & 66.1 & 63.7 & 64.3 & 72.1 & 74.3 & 76.1 & 74.5 & 73.8 & 86.6 \\
\hline$m$ & 19.5 & 22.5 & 42.5 & 55.2 & 49.6 & 55.8 & 64.3 & 62.9 & 65.2 & 64.4 & 62.9 & 69.3 & 88.8 \\
\hline
\end{tabular}




\begin{tabular}{|c|c|c|c|c|c|c|c|c|c|}
\hline \multirow{2}{*}{$\begin{array}{l}\Delta m \\
(\mathrm{GeV})\end{array}$} & \multicolumn{9}{|c|}{$m_{\tilde{\chi}_{1}^{ \pm}}(\mathrm{GeV})$} \\
\hline & 50 & 60 & 70 & 80 & 85 & 90 & 94 & 99 & 102 \\
\hline \multicolumn{10}{|c|}{ General Selection Efficiency ( \% ) } \\
\hline 2 & $5.3 \pm 0.4$ & $3.9 \pm 0.3$ & $2.6 \pm 0.3$ & $1.5 \pm 0.2$ & $1.0 \pm 0.2$ & $0.7 \pm 0.1$ & $0.4 \pm 0.1$ & $0.2 \pm 0.1$ & $0.1 \pm 0.0$ \\
\hline 3 & $16.2 \pm 0.6$ & $15.9 \pm 0.6$ & $14.3 \pm 0.6$ & $12.6 \pm 0.5$ & $11.7 \pm 0.5$ & $10.5 \pm 0.5$ & $9.3 \pm 0.5$ & $8.1 \pm 0.4$ & $7.6 \pm 0.4$ \\
\hline 4 & $26.5 \pm 0.8$ & $27.4 \pm 0.7$ & $24.6 \pm 0.7$ & $24.3 \pm 0.7$ & $23.9 \pm 0.7$ & $22.5 \pm 0.7$ & $20.9 \pm 0.6$ & $20.0 \pm 0.6$ & $20.0 \pm 0.6$ \\
\hline 5 & $35.6 \pm 0.8$ & $35.2 \pm 0.8$ & $33.8 \pm 0.8$ & $33.8 \pm 0.7$ & $33.8 \pm 0.7$ & $33.1 \pm 0.7$ & $31.8 \pm 0.7$ & $30.9 \pm 0.7$ & $31.0 \pm 0.7$ \\
\hline 10 & $58.1 \pm 0.9$ & $59.6 \pm 0.8$ & $59.1 \pm 0.8$ & $58.5 \pm 0.8$ & $57.6 \pm 0.8$ & $56.3 \pm 0.8$ & $55.5 \pm 0.8$ & $55.1 \pm 0.8$ & $55.1 \pm 0.8$ \\
\hline 20 & $0.0 \pm 0.0$ & $76.1 \pm 0.7$ & $76.8 \pm 0.7$ & $76.2 \pm 0.7$ & $75.7 \pm 0.7$ & $75.4 \pm 0.7$ & $75.3 \pm 0.7$ & $75.5 \pm 0.7$ & $75.2 \pm 0.7$ \\
\hline$(m-15) / 2$ & $0.0 \pm 0.0$ & $77.6 \pm 0.7$ & $81.4 \pm 0.6$ & $84.1 \pm 0.6$ & $84.9 \pm 0.6$ & $85.2 \pm 0.6$ & $85.8 \pm 0.6$ & $87.2 \pm 0.5$ & $88.1 \pm 0.5$ \\
\hline$m-35$ & $68.5 \pm 0.8$ & $78.6 \pm 0.7$ & $83.3 \pm 0.6$ & $85.9 \pm 0.6$ & $87.2 \pm 0.5$ & $88.3 \pm 0.5$ & $89.1 \pm 0.5$ & $90.3 \pm 0.5$ & $90.6 \pm 0.5$ \\
\hline \multicolumn{10}{|c|}{ 95\% CL upper limit on cross-section times $B R^{2}\left(\tilde{\chi}_{1}^{ \pm} \rightarrow \ell^{ \pm} \tilde{\nu}\right)(\mathrm{fb})$} \\
\hline 2 & 104 & 133 & 218 & 473 & 835 & 1960 & 3390 & 8230 & - \\
\hline 3 & 41.7 & 41.1 & 42.0 & 52.7 & 63.9 & 73.4 & 88.1 & 135 & 350 \\
\hline 4 & 26.0 & 24.5 & 29.1 & 25.6 & 28.8 & 29.4 & 36.1 & 52.3 & 129 \\
\hline 5 & 20.2 & 19.5 & 19.7 & 21.3 & 22.7 & 24.6 & 34.1 & 34.5 & 84.1 \\
\hline 10 & 29.3 & 24.2 & 20.2 & 17.1 & 16.2 & 17.0 & 21.2 & 25.6 & 48.5 \\
\hline & - & 30.2 & 27.4 & 23.5 & 25.3 & 19.9 & 19.5 & 21.2 & 40.1 \\
\hline$(m-15) / 2$ & - & 33.1 & 34.9 & 35.9 & 45.2 & 47.9 & 70.9 & 78.8 & 64.7 \\
\hline$m-35$ & 65.1 & 42.0 & 45.3 & 65.6 & 73.0 & 69.3 & 77.3 & 156 & 273 \\
\hline \multicolumn{10}{|c|}{$\overline{\text { expected upper limit on cross-section times } B R^{2}\left(\tilde{\chi}_{1}^{ \pm} \rightarrow \ell^{ \pm} \tilde{\nu}\right)(\mathrm{fb})}$} \\
\hline 2 & 111 & 156 & 250 & 503 & 791 & 1720 & 3000 & 9900 & - \\
\hline 3 & 49.0 & 47.6 & 50.3 & 56.5 & 61.9 & 74.8 & 106 & 174 & 401 \\
\hline 4 & 34.6 & 33.2 & 34.4 & 32.4 & 34.3 & 38.5 & 51.0 & 72.9 & 150 \\
\hline 5 & 30.5 & 28.6 & 28.2 & 27.5 & 28.0 & 29.6 & 37.0 & 53.1 & 105 \\
\hline 10 & 35.7 & 29.7 & 26.3 & 23.5 & 23.3 & 24.1 & 28.4 & 36.8 & 71.4 \\
\hline & - & 53.8 & 44.7 & 38.5 & 34.9 & 33.1 & 37.4 & 45.5 & 72.3 \\
\hline$(m-15) / 2$ & - & 59.1 & 61.6 & 64.0 & 68.6 & 77.2 & 85.6 & 89.0 & 117 \\
\hline$m-35$ & 98.9 & 66.1 & 77.8 & 90.2 & 98.9 & 97.5 & 100 & 101 & 136 \\
\hline \multicolumn{10}{|c|}{ CL for consistency with SM (\%) } \\
\hline 2 & 46.7 & 66.5 & 60.5 & 49.1 & 35.6 & 25.8 & 32.0 & 100.0 & - \\
\hline 3 & 61.3 & 60.6 & 66.3 & 54.1 & 37.8 & 43.6 & 75.4 & 100.0 & 100.0 \\
\hline 4 & 76.7 & 76.5 & 64.7 & 68.7 & 63.6 & 77.0 & 81.4 & 100.0 & 100.0 \\
\hline 5 & 85.7 & 83.9 & 83.4 & 72.3 & 68.3 & 65.3 & 54.8 & 86.2 & 100.0 \\
\hline 10 & 66.0 & 66.6 & 72.9 & 78.0 & 81.6 & 81.2 & 76.4 & 83.5 & 92.2 \\
\hline & - & 95.4 & 91.9 & 90.9 & 79.0 & 91.7 & 97.6 & 99.6 & 99.6 \\
\hline$(m-15) / 2$ & - & 96.4 & 94.8 & 94.8 & 88.1 & 90.7 & 67.2 & 57.7 & 96.1 \\
\hline$m-35$ & 86.3 & 91.1 & 94.6 & 79.5 & 78.9 & 82.9 & 72.8 & 7.5 & 0.9 \\
\hline
\end{tabular}




\begin{tabular}{|c|c|c|c|c|c|c|c|c|c|c|}
\hline \multirow{2}{*}{$\begin{array}{l}\Delta m \\
(\mathrm{GeV})\end{array}$} & \multicolumn{10}{|c|}{$m_{\tilde{\chi}_{1}^{ \pm}}(\mathrm{GeV})$} \\
\hline & 50 & 60 & 70 & 80 & 85 & 90 & 94 & 99 & 102 & 103 \\
\hline \multicolumn{11}{|c|}{$\begin{array}{l}\text { General Selection Efficiency (\%) } \\
\end{array}$} \\
\hline 3 & $1.7 \pm 0.2$ & $1.5 \pm 0.2$ & $1.4 \pm 0.2$ & $0.8 \pm 0.1$ & $0.4 \pm 0.1$ & $0.4 \pm 0.1$ & $0.3 \pm 0.1$ & $\overline{0.1 \pm 0.0}$ & $\overline{0.0 \pm 0.0}$ & $0.0 \pm 0.0$ \\
\hline 5 & $11.2 \pm 0.5$ & $10.4 \pm 0.5$ & $10.2 \pm 0.5$ & $9.1 \pm 0.5$ & $7.3 \pm 0.4$ & $6.8 \pm 0.4$ & $6.0 \pm 0.4$ & $5.6 \pm 0.4$ & $4.8 \pm 0.3$ & $4.4 \pm 0.3$ \\
\hline 10 & $32.6 \pm 0.7$ & $34.2 \pm 0.8$ & $33.5 \pm 0.7$ & $32.4 \pm 0.7$ & $31.0 \pm 0.7$ & $32.5 \pm 0.7$ & $31.2 \pm 0.7$ & $29.3 \pm 0.7$ & $29.5 \pm 0.7$ & $29.7 \pm 0.7$ \\
\hline 20 & $52.7 \pm 0.8$ & $53.7 \pm 0.8$ & $54.8 \pm 0.8$ & $54.8 \pm 0.8$ & $55.4 \pm 0.8$ & $55.0 \pm 0.8$ & $55.2 \pm 0.8$ & $53.4 \pm 0.8$ & $54.6 \pm 0.8$ & $55.4 \pm 0.8$ \\
\hline$m / 2$ & $57.5 \pm 0.8$ & $63.9 \pm 0.8$ & $69.5 \pm 0.7$ & $75.7 \pm 0.7$ & $74.9 \pm 0.7$ & $76.6 \pm 0.7$ & $77.2 \pm 0.7$ & $78.0 \pm 0.7$ & $78.4 \pm 0.7$ & $77.9 \pm 0.7$ \\
\hline$m-20$ & $62.2 \pm 0.8$ & $71.9 \pm 0.7$ & $75.8 \pm 0.7$ & $79.2 \pm 0.6$ & $82.4 \pm 0.6$ & $82.9 \pm 0.6$ & $84.6 \pm 0.6$ & $87.2 \pm 0.5$ & $88.5 \pm 0.5$ & $88.8 \pm 0.5$ \\
\hline$m-10$ & $68.9 \pm 0.7$ & $73.4 \pm 0.7$ & $79.2 \pm 0.6$ & $82.2 \pm 0.6$ & $84.3 \pm 0.6$ & $87.8 \pm 0.5$ & $89.0 \pm 0.5$ & $90.2 \pm 0.5$ & $90.4 \pm 0.5$ & $90.3 \pm 0.5$ \\
\hline$m$ & $72.2 \pm 0.7$ & $77.2 \pm 0.7$ & $80.4 \pm 0.6$ & $85.2 \pm 0.6$ & $87.3 \pm 0.5$ & $89.2 \pm 0.5$ & $89.7 \pm 0.5$ & $89.6 \pm 0.5$ & $90.5 \pm 0.5$ & $91.1 \pm 0.5$ \\
\hline \multicolumn{11}{|c|}{$\overline{95 \%}$ CL upper limit on cross-section times $B R^{2}\left(\tilde{\chi}_{1}^{ \pm} \rightarrow \ell^{ \pm} \nu \tilde{\chi}_{1}^{0}\right)(\mathrm{fb})$} \\
\hline 3 & 190 & 249 & 374 & 979 & 1170 & 1810 & 4180 & 10400 & & \\
\hline 5 & 59.0 & 62.9 & 55.9 & 80.0 & 86.1 & 106 & 151 & 195 & 565 & 7640 \\
\hline 10 & 29.8 & 23.5 & 21.8 & 24.1 & 23.7 & 27.7 & 36.1 & 46.3 & 87.4 & 1130 \\
\hline 20 & 33.6 & 27.9 & 25.3 & 19.7 & 19.1 & 19.5 & 22.7 & 26.8 & 54.3 & 605 \\
\hline$m / 2$ & 37.2 & 32.1 & 33.2 & 27.8 & 30.1 & 34.2 & 45.4 & 37.5 & 56.5 & 441 \\
\hline$m-20$ & 43.3 & 39.8 & 36.9 & 40.3 & 43.1 & 56.2 & 77.0 & 108 & 151 & 1050 \\
\hline$m-10$ & 50.5 & 45.4 & 45.4 & 47.6 & 56.7 & 68.2 & 98.4 & 155 & 248 & 1230 \\
\hline$m$ & 58.4 & 52.6 & 58.7 & 66.6 & 92.5 & 93.5 & 105 & 175 & 264 & 1180 \\
\hline \multicolumn{11}{|c|}{ expected upper limit on cross-section times $B R^{2}\left(\tilde{\chi}_{1}^{ \pm} \rightarrow \ell^{ \pm} \nu \tilde{\chi}_{1}^{0}\right)(\mathrm{fb})$} \\
\hline 3 & 270 & 334 & 470 & 883 & 1360 & 2490 & 3960 & 12900 & & \\
\hline 5 & 67.5 & 67.9 & 73.5 & 83.3 & 99.0 & 114 & 159 & 259 & 664 & 7670 \\
\hline 10 & 36.4 & 33.5 & 32.5 & 32.5 & 33.0 & 35.8 & 43.0 & 60.2 & 117 & 1160 \\
\hline 20 & 41.1 & 35.5 & 31.5 & 29.8 & 30.0 & 30.6 & 36.1 & 47.4 & 84.3 & 662 \\
\hline$m / 2$ & 46.1 & 46.6 & 43.9 & 43.3 & 43.8 & 48.9 & 59.9 & 81.2 & 131 & 695 \\
\hline$m-20$ & 50.9 & 54.3 & 56.2 & 61.5 & 70.0 & 80.9 & 102 & 128 & 189 & 833 \\
\hline$m-10$ & 61.2 & 63.6 & 68.4 & 75.7 & 85.1 & 101 & 120 & 130 & 193 & 835 \\
\hline$m$ & 70.1 & 71.2 & 77.5 & 98.1 & 114 & 114 & 120 & 133 & 187 & 839 \\
\hline \multicolumn{11}{|c|}{ CL for consistency with SM (\%) } \\
\hline 3 & $\begin{array}{l}83.8 \\
\end{array}$ & 77.6 & $\begin{array}{l}72.4 \\
\end{array}$ & 29.0 & 59.5 & 86.6 & 33.5 & 100.0 & & \\
\hline 5 & 59.6 & 50.9 & 74.3 & 47.3 & 60.5 & 49.8 & 47.3 & 100.0 & 100.0 & 100.0 \\
\hline 10 & 66.3 & 82.8 & 86.0 & 76.4 & 80.2 & 71.8 & 61.6 & 73.0 & 100.0 & 100.0 \\
\hline 20 & 66.3 & 72.6 & 67.2 & 86.3 & 88.3 & 88.4 & 90.6 & 95.7 & 90.7 & 100.0 \\
\hline$m / 2$ & 70.3 & 81.2 & 73.8 & 88.1 & 82.8 & 81.2 & 72.5 & 99.5 & 99.8 & 96.3 \\
\hline$m-20$ & 62.7 & 77.1 & 86.8 & 87.0 & 92.4 & 81.8 & 76.7 & 64.1 & 68.6 & 20.8 \\
\hline$m-10$ & 64.4 & 80.3 & 86.9 & 90.6 & 88.0 & 86.5 & 68.5 & 23.5 & 17.3 & 8.8 \\
\hline$m$ & 64.5 & 75.9 & 76.7 & 86.2 & 69.9 & 67.8 & 59.3 & 15.6 & 11.3 & 10.9 \\
\hline
\end{tabular}




\begin{tabular}{|c|c|c|c|c|c|c|c|c|c|c|c|}
\hline \multicolumn{12}{|c|}{$m_{\mathrm{H}}+(\mathrm{GeV})$} \\
\hline 50 & 55 & 60 & 65 & 70 & 75 & 80 & 85 & 90 & 94 & 99 & 103 \\
\hline \multicolumn{12}{|c|}{ General Selection Efficiency ( \% ) } \\
\hline $68.8 \pm 0.7$ & $70.1 \pm 0.6$ & $71.9 \pm 0.6$ & $75.1 \pm 0.6$ & $75.3 \pm 0.6$ & $76.9 \pm 0.6$ & $76.6 \pm 0.6$ & $78.9 \pm 0.6$ & $78.6 \pm 0.6$ & $79.9 \pm 0.6$ & $80.2 \pm 0.6$ & $80.2 \pm 0.6$ \\
\hline \multicolumn{12}{|c|}{$95 \%$ CL upper limit on cross-section times $B R^{2}\left(\mathrm{H}^{ \pm} \rightarrow \tau^{ \pm} \nu_{\tau}\right)(\mathrm{fb})$} \\
\hline 63.8 & 57.4 & 50.9 & 55.4 & 52.2 & 47.0 & 48.1 & 52.4 & 61.9 & 70.8 & 99.5 & 352 \\
\hline \multicolumn{12}{|c|}{ expected upper limit on cross-section times $B R^{2}\left(\mathrm{H}^{ \pm} \rightarrow \tau^{ \pm} \nu_{\tau}\right)(\mathrm{fb})$} \\
\hline 53.0 & 53.5 & 54.4 & 57.3 & 57.4 & 59.7 & 62.9 & 68.1 & 78.1 & 88.9 & 117 & 539 \\
\hline \multicolumn{12}{|c|}{ CL for consistency with SM (\%) } \\
\hline 23.8 & 34.9 & 50.5 & 48.1 & 54.4 & 71.3 & 74.8 & 75.1 & 72.2 & 68.2 & 62.2 & 87.3 \\
\hline
\end{tabular}




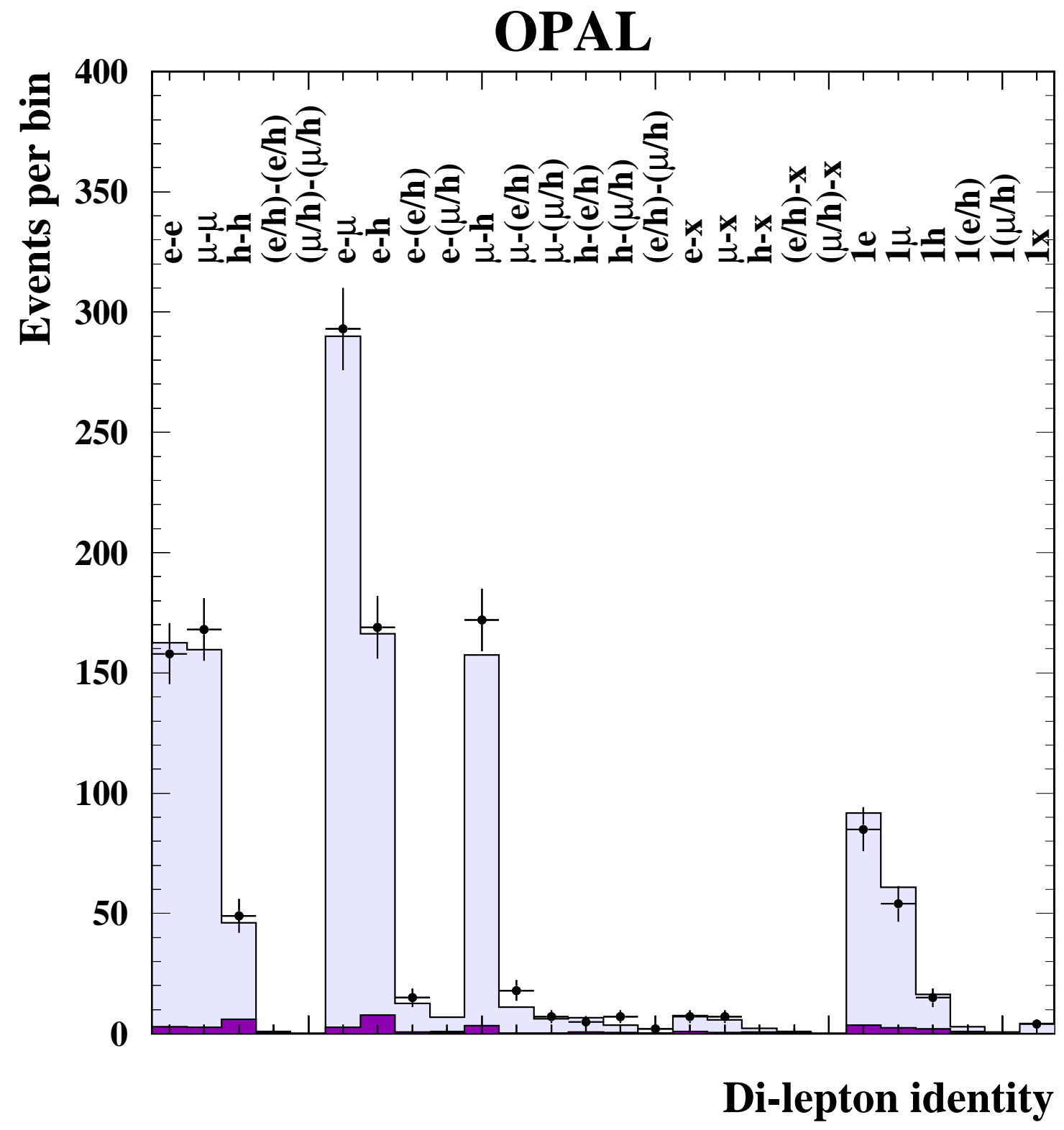

Figure 1: Di-lepton identities for the data at $\sqrt{s}=189-208 \mathrm{GeV}$ compared with Standard Model expectations. The data are shown as the points with error bars (statistical errors only). The Standard Model Monte Carlo prediction dominated by 4-fermion processes with genuine prompt missing energy and momentum $\left(\ell^{+} \nu \ell^{-} \bar{\nu}\right)$ is shown as the lightly shaded histogram and the background component, arising mainly from processes with four charged leptons in the final state, is shown as the darkly shaded histogram. The Standard Model Monte Carlo histograms are normalized to the integrated luminosity of the data. The last six bins correspond to "single-lepton events". 


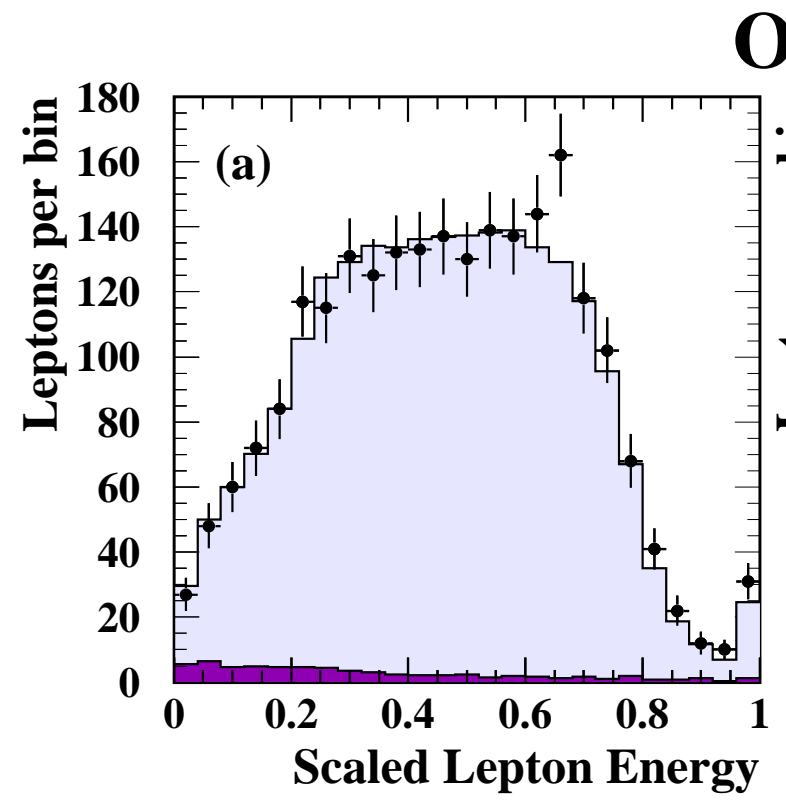

OPAL
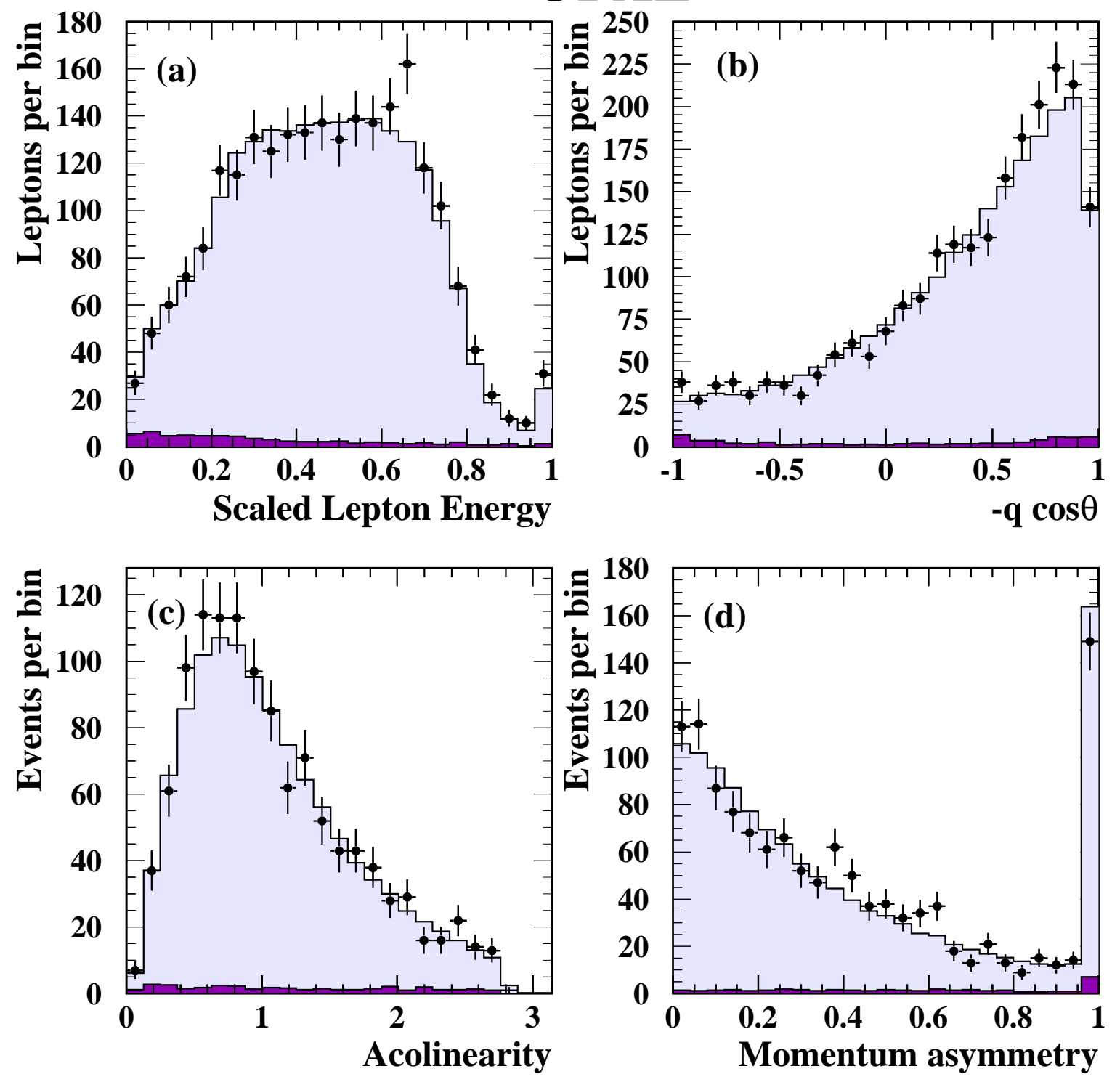

Figure 2: Distributions of (a) the lepton momentum divided by the beam energy, (b) $-q \cos \theta$, (c) acolinearity (in radians) and (d) momentum asymmetry for the event sample produced by the general selection at $\sqrt{s}=189-208 \mathrm{GeV}$. The data are shown as the points with error bars (statistical errors only). The Standard Model Monte Carlo prediction dominated by 4fermion processes with genuine prompt missing energy and momentum $\left(\ell^{+} \nu \ell^{-} \bar{\nu}\right)$ is shown as the lightly shaded histogram and the background component, arising mainly from processes with four charged leptons in the final state, is shown as the darkly shaded histogram. The Standard Model Monte Carlo histograms are normalized to the integrated luminosity of the data. In (a) and (b) there are two entries per event for events containing two identified leptons. 


\section{OPAL}
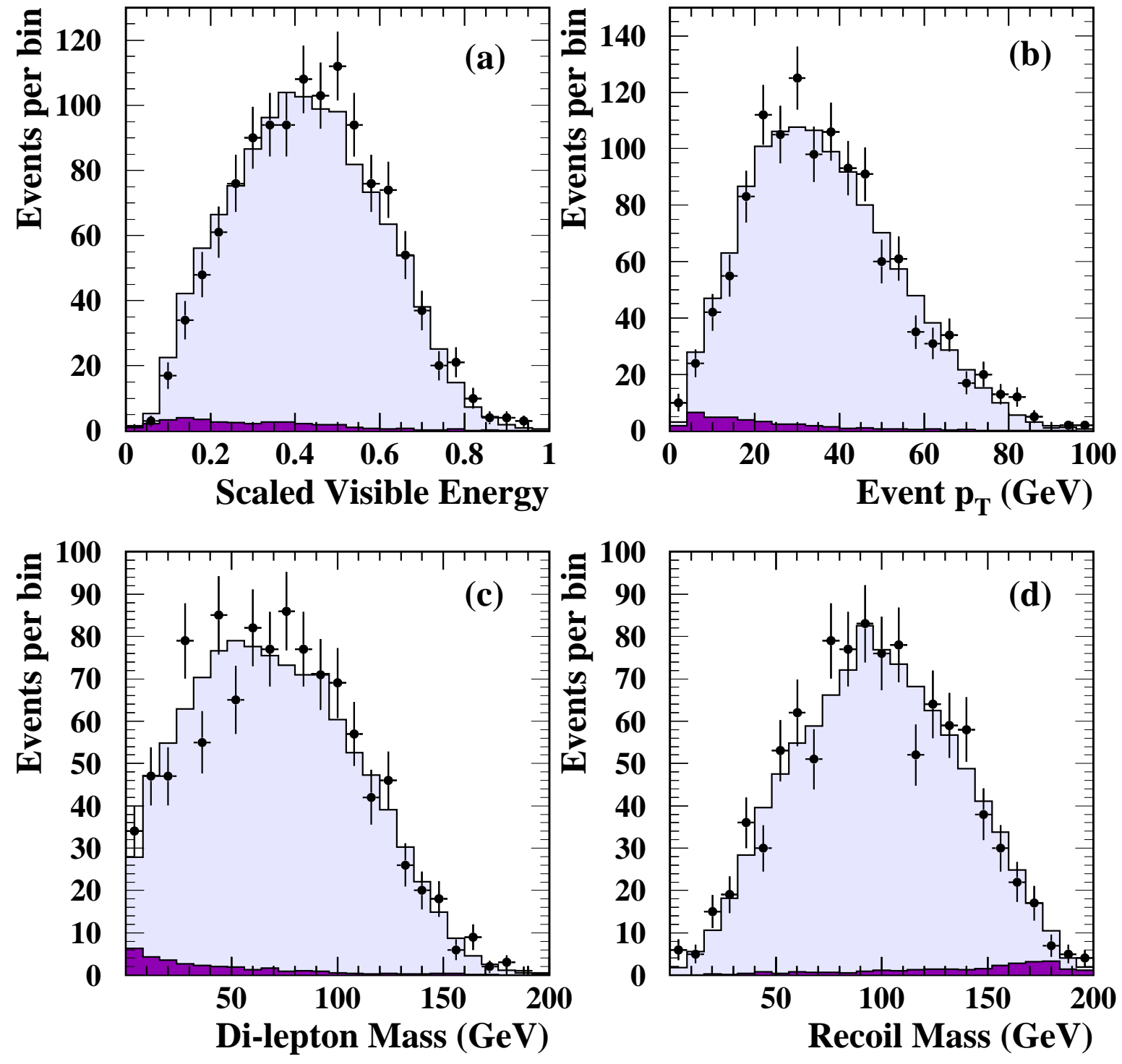

Figure 3: Distributions of (a) the total visible energy divided by the centre-of-mass energy, (b) the net transverse momentum of the event (c) di-lepton mass and (d) recoil mass to the di-lepton system for the event sample produced by the general selection at $\sqrt{s}=189-208 \mathrm{GeV}$. The data are shown as the points with error bars (statistical errors only). The Standard Model Monte Carlo prediction dominated by 4-fermion processes with genuine prompt missing energy and momentum $\left(\ell^{+} \nu \ell^{-} \bar{\nu}\right)$ is shown as the lightly shaded histogram and the background component, arising mainly from processes with four charged leptons in the final state, is shown as the darkly shaded histogram. The Standard Model Monte Carlo histograms are normalized to the integrated luminosity of the data. 


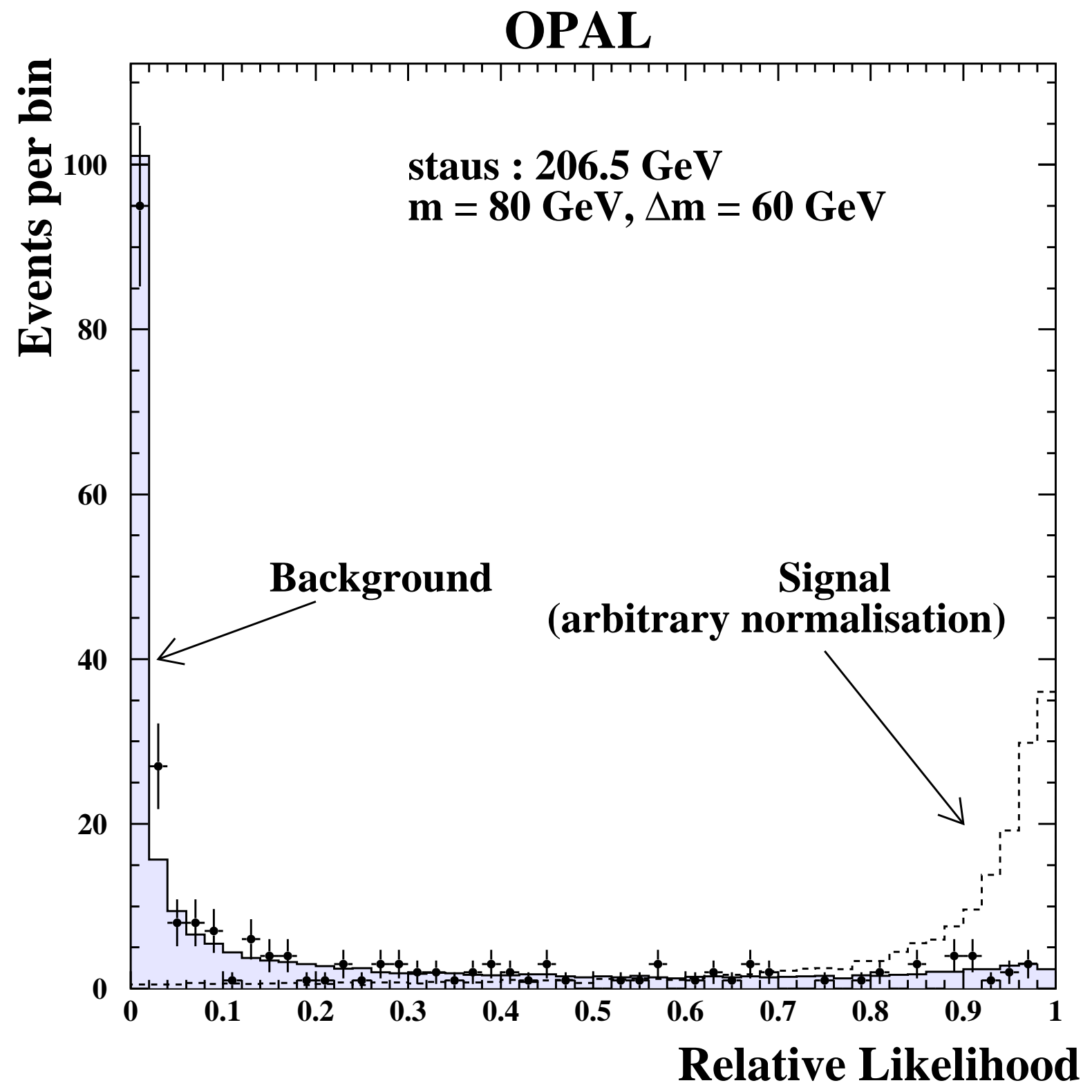

Figure 4: Distributions of the relative likelihood, $L_{\mathrm{R}}$, for Standard Model Monte Carlo (shaded histogram), signal (open histogram) and data (points with error bars, statistical errors only), in the analysis for staus with a mass of $80 \mathrm{GeV}$ for a stau-neutralino mass difference of $60 \mathrm{GeV}$ for the $\sqrt{s}=206.5 \mathrm{GeV}$ centre-of-mass energy bin. The Standard Model Monte Carlo histogram is normalized to the integrated luminosity of the data. 


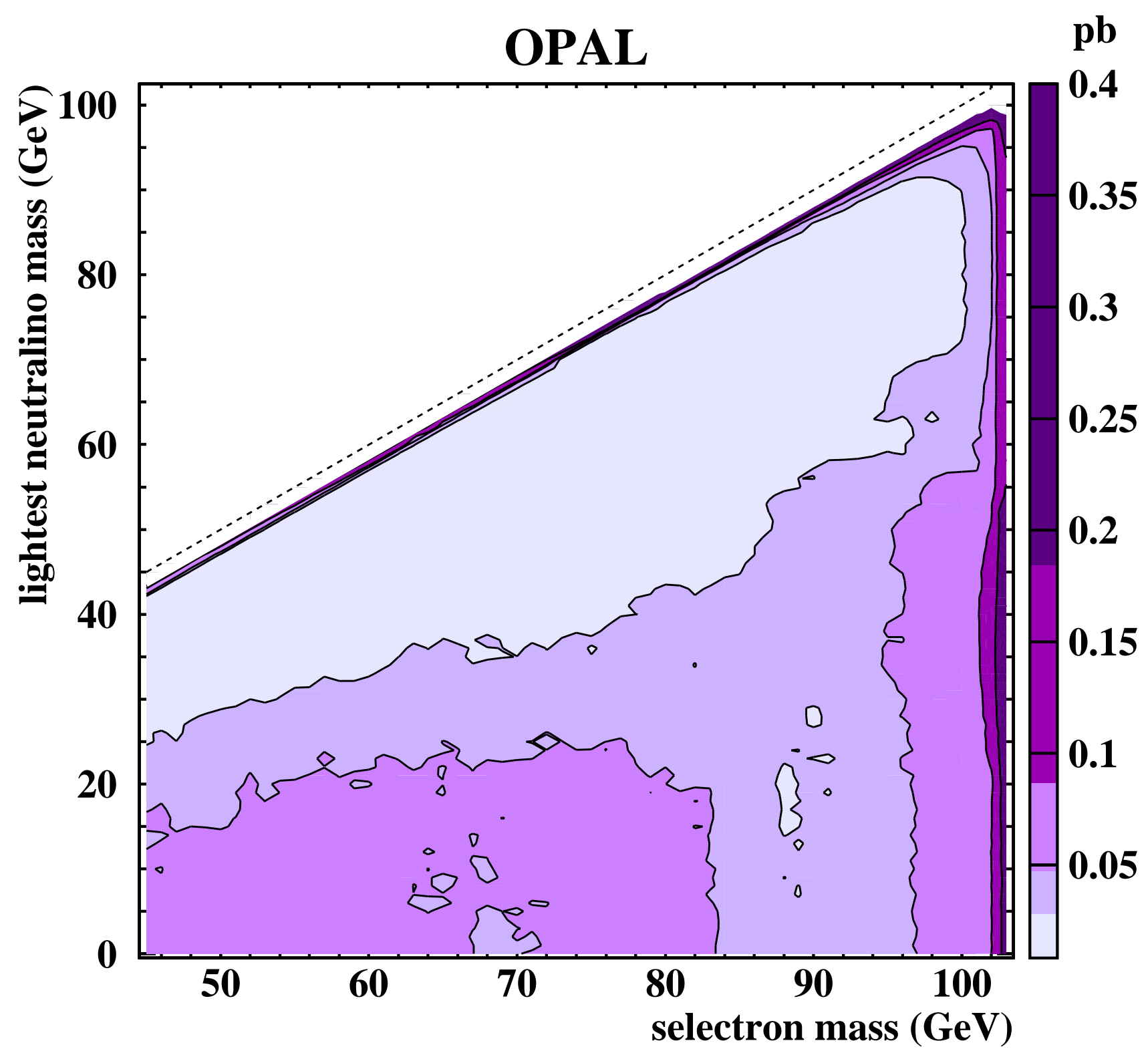

Figure 5: Contours of the 95\% CL upper limits on the selectron pair cross-section times $B R^{2}\left(\tilde{\mathrm{e}} \rightarrow \mathrm{e} \tilde{\chi}_{1}^{0}\right)$ at $208 \mathrm{GeV}$ based on combining the 183 - $208 \mathrm{GeV}$ data-sets assuming a $\beta^{3} / s$ dependence of the cross-section. The kinematically allowed region lies below the dashed line. The unshaded region at very low $\Delta m$ is experimentally inaccessible in this search. 
OPAL

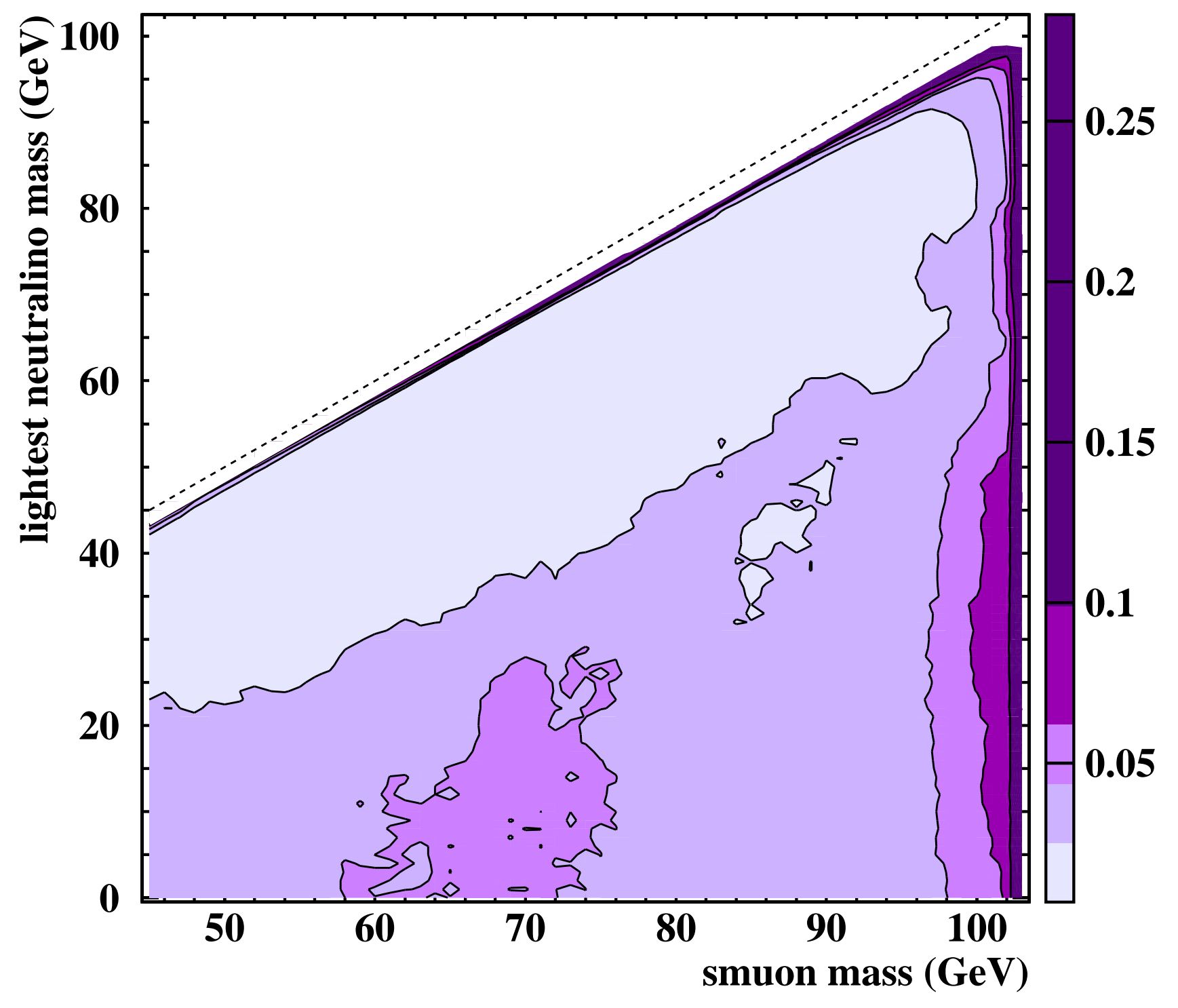

Figure 6: Contours of the 95\% CL upper limits on the smuon pair cross-section times $B R^{2}(\tilde{\mu} \rightarrow$ $\left.\mu \tilde{\chi}_{1}^{0}\right)$ at $208 \mathrm{GeV}$ based on combining the 183 - $208 \mathrm{GeV}$ data-sets assuming a $\beta^{3} / \mathrm{s}$ dependence of the cross-section. The kinematically allowed region lies below the dashed line. The unshaded region at very low $\Delta m$ is experimentally inaccessible in this search. 
OPAL

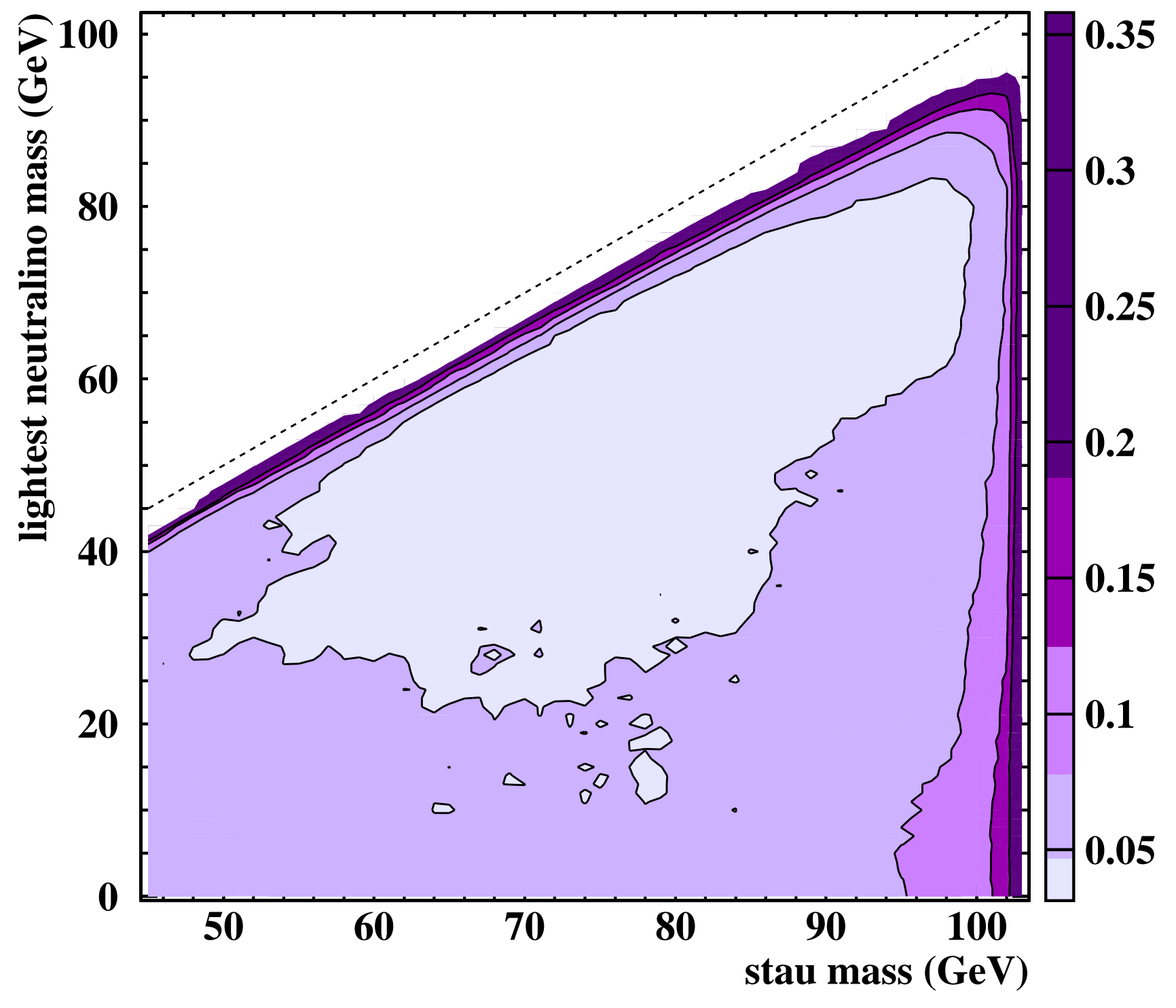

Figure 7: Contours of the 95\% CL upper limits on the stau pair cross-section times $B R^{2}(\tilde{\tau} \rightarrow$ $\tau \tilde{\chi}_{1}^{0}$ ) at $208 \mathrm{GeV}$ based on combining the 183 - $208 \mathrm{GeV}$ data-sets assuming a $\beta^{3} / \mathrm{s}$ dependence of the cross-section. The kinematically allowed region lies below the dashed line. The unshaded region at very low $\Delta m$ is experimentally inaccessible in this search. 
OPAL

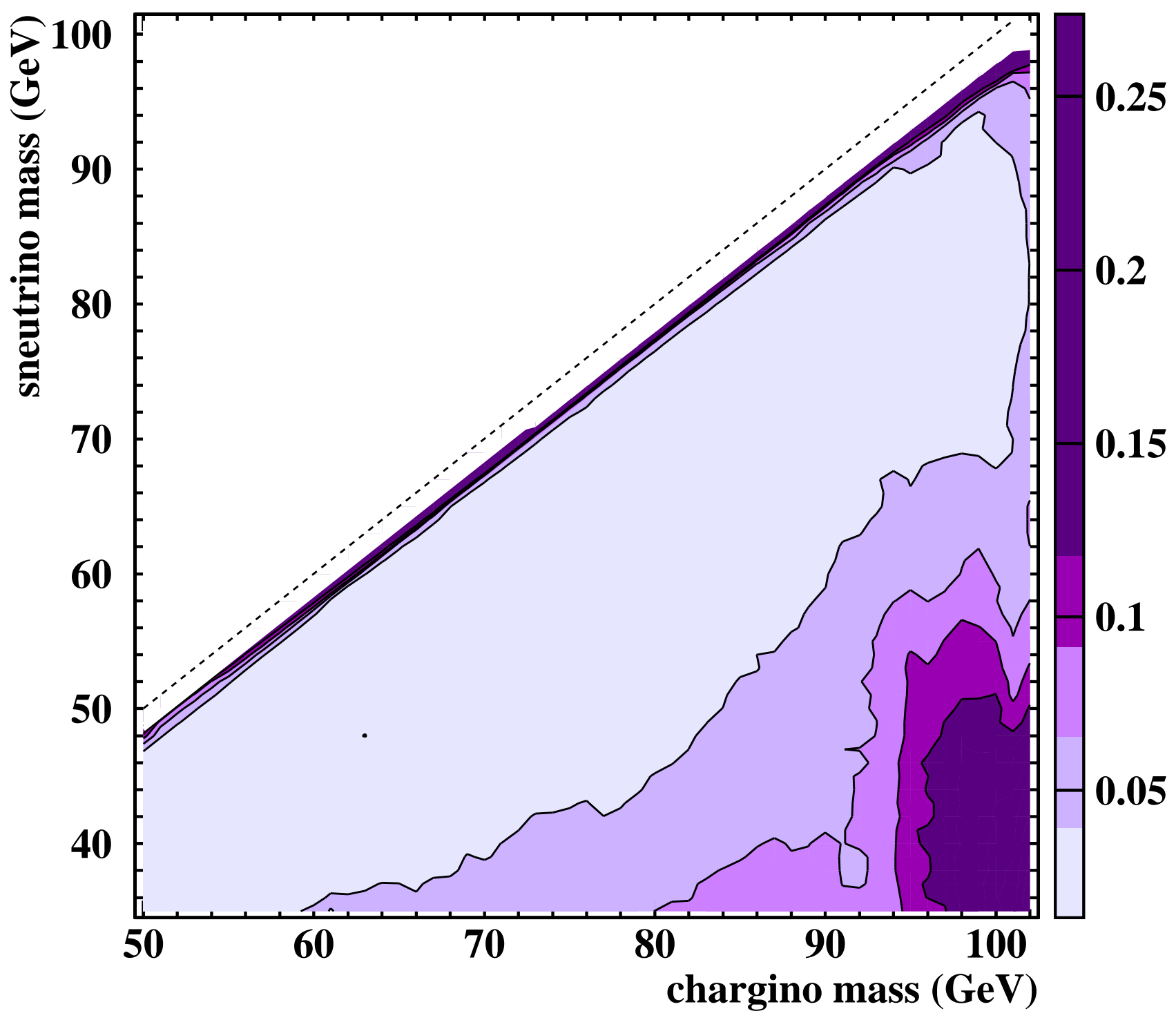

Figure 8: Contours of the 95\% CL upper limits on the chargino pair cross-section times branching ratio squared for $\tilde{\chi}_{1}^{ \pm} \rightarrow \ell^{ \pm} \tilde{\nu}$ (2-body decay) at $\sqrt{s}=208 \mathrm{GeV}$. The limits have been calculated for the case where the three sneutrino generations are mass degenerate. Only sneutrino masses above $35 \mathrm{GeV}$ have been considered given constraints from the $\mathrm{Z}^{0}$ line-shape. The limit is obtained by combining the $183-208 \mathrm{GeV}$ data-sets assuming a $\beta / s$ dependence of the crosssection. The kinematically allowed region lies below the dashed line. The unshaded region at very low $\Delta m$ is experimentally inaccessible in this search. 
OPAL

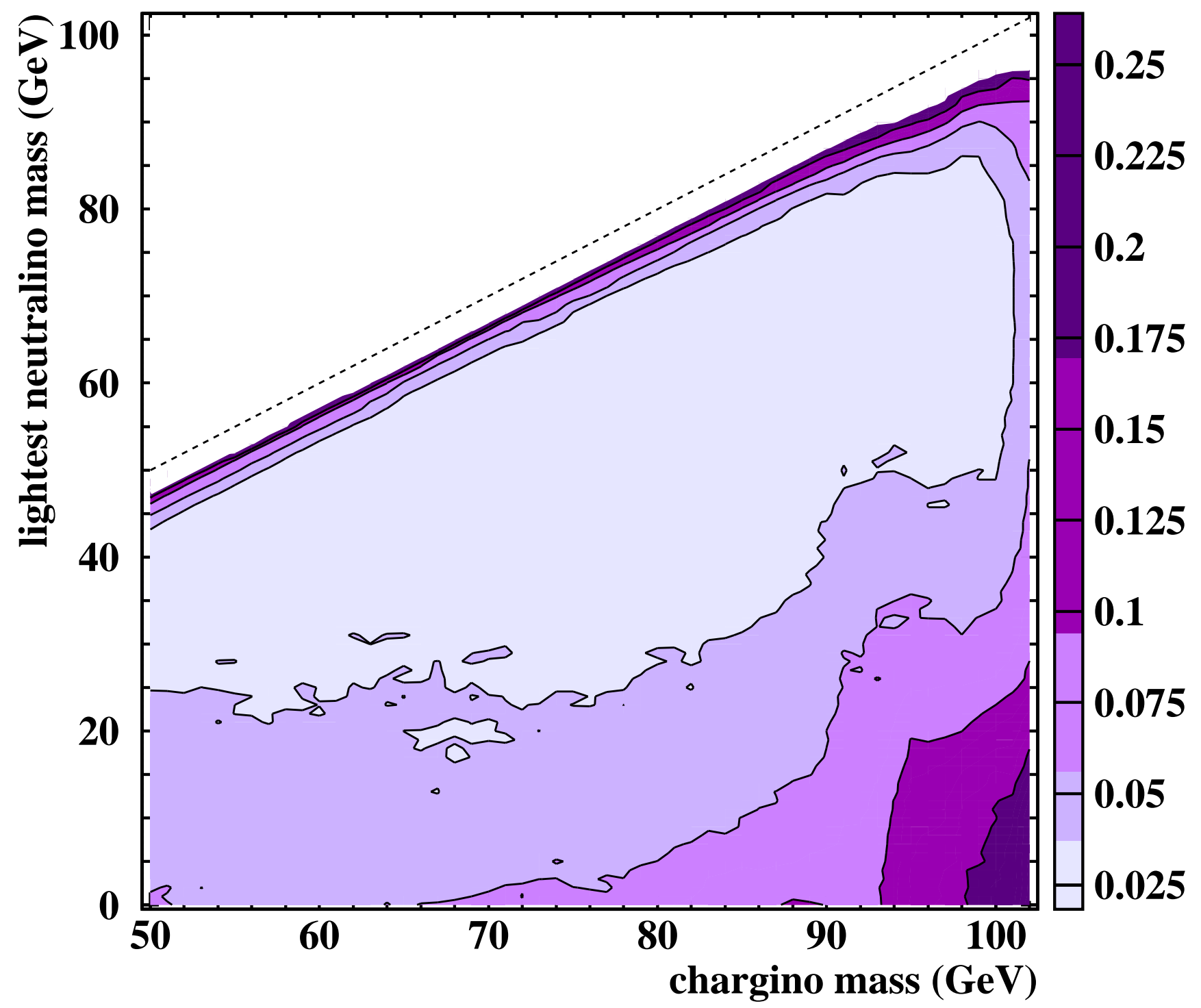

Figure 9: Contours of the 95\% CL upper limits on the chargino pair cross-section times branching ratio squared for $\tilde{\chi}_{1}^{ \pm} \rightarrow \ell^{ \pm} \nu \tilde{\chi}_{1}^{0}$ (3-body decay) at $\sqrt{s}=208 \mathrm{GeV}$. The limit is obtained by combining the $183-208 \mathrm{GeV}$ data-sets assuming a $\beta / s$ dependence of the cross-section. The kinematically allowed region lies below the dashed line. The unshaded region at very low $\Delta m$ is experimentally inaccessible in this search. 


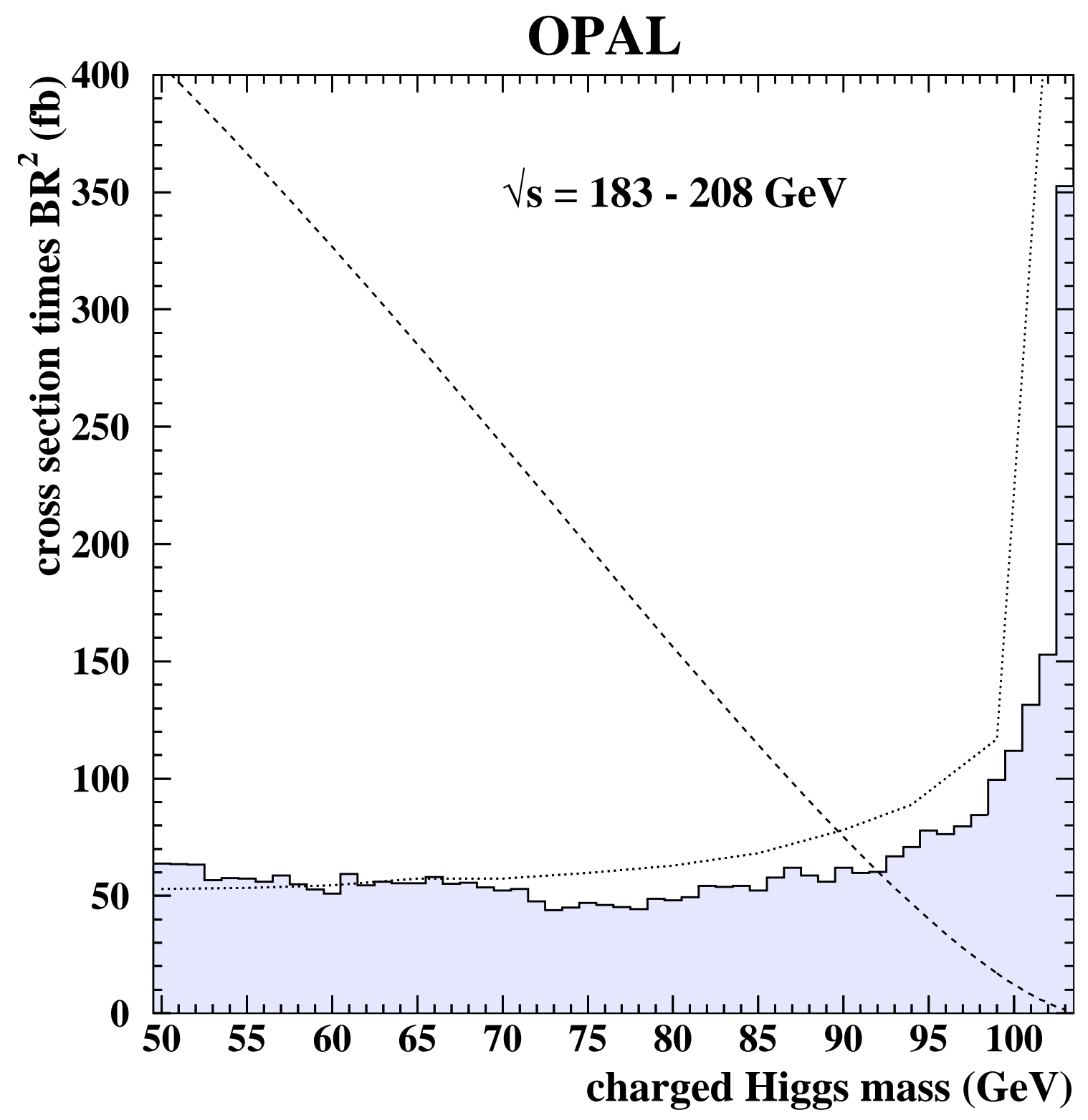

Figure 10: The solid histogram shows the 95\% CL upper limit on the charged Higgs pair production cross-section times branching ratio squared for the decay $\mathrm{H}^{ \pm} \rightarrow \tau^{ \pm} \nu_{\tau}$ at $\sqrt{s}=208 \mathrm{GeV}$. The limit is obtained by combining the 183 - $208 \mathrm{GeV}$ data-sets assuming a $\beta^{3} / \mathrm{s}$ dependence of the cross-section. For comparison, the dashed curve shows the prediction from HZHA at $\sqrt{s}=208 \mathrm{GeV}$ assuming a $100 \%$ branching ratio for the decay $\mathrm{H}^{ \pm} \rightarrow \tau^{ \pm} \nu_{\tau}$. The expected limit calculated from Standard Model Monte Carlo alone is indicated by the dotted line. 
OPAL

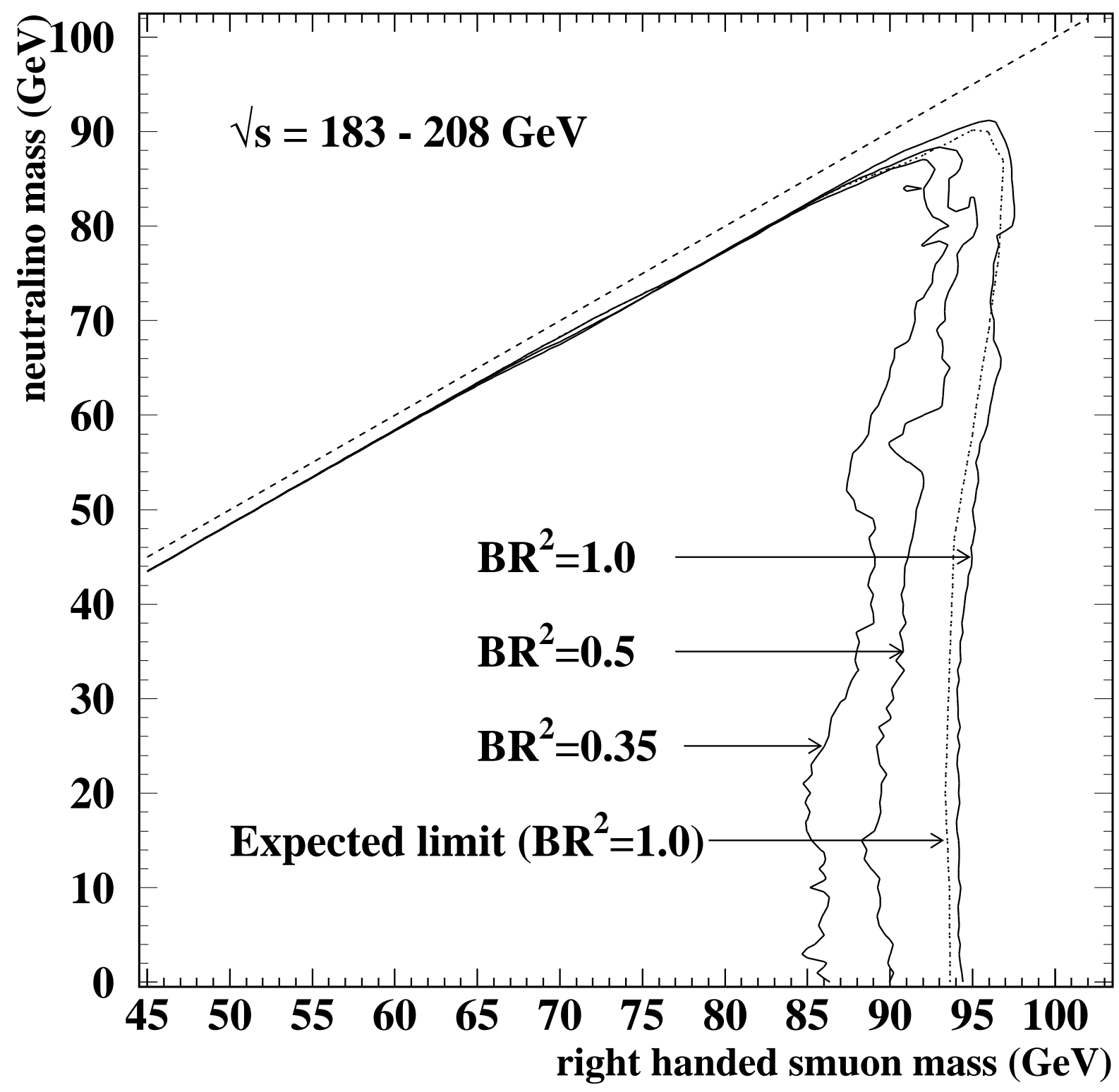

Figure 11: 95\% CL exclusion region for right-handed smuon pair production obtained by combining the $\sqrt{s}=183-208 \mathrm{GeV}$ data-sets. The limits are calculated for several values of the branching ratio squared for $\tilde{\mu}_{R}^{ \pm} \rightarrow \mu^{ \pm} \tilde{\chi}_{1}^{0}$ that are indicated in the figure. Otherwise they have no supersymmetry model assumptions. The kinematically allowed region lies below the dashed line. The expected limit for $B R^{2}=1.0$, calculated from Monte Carlo alone, is indicated by the dash-dotted line. 


\section{OPAL}

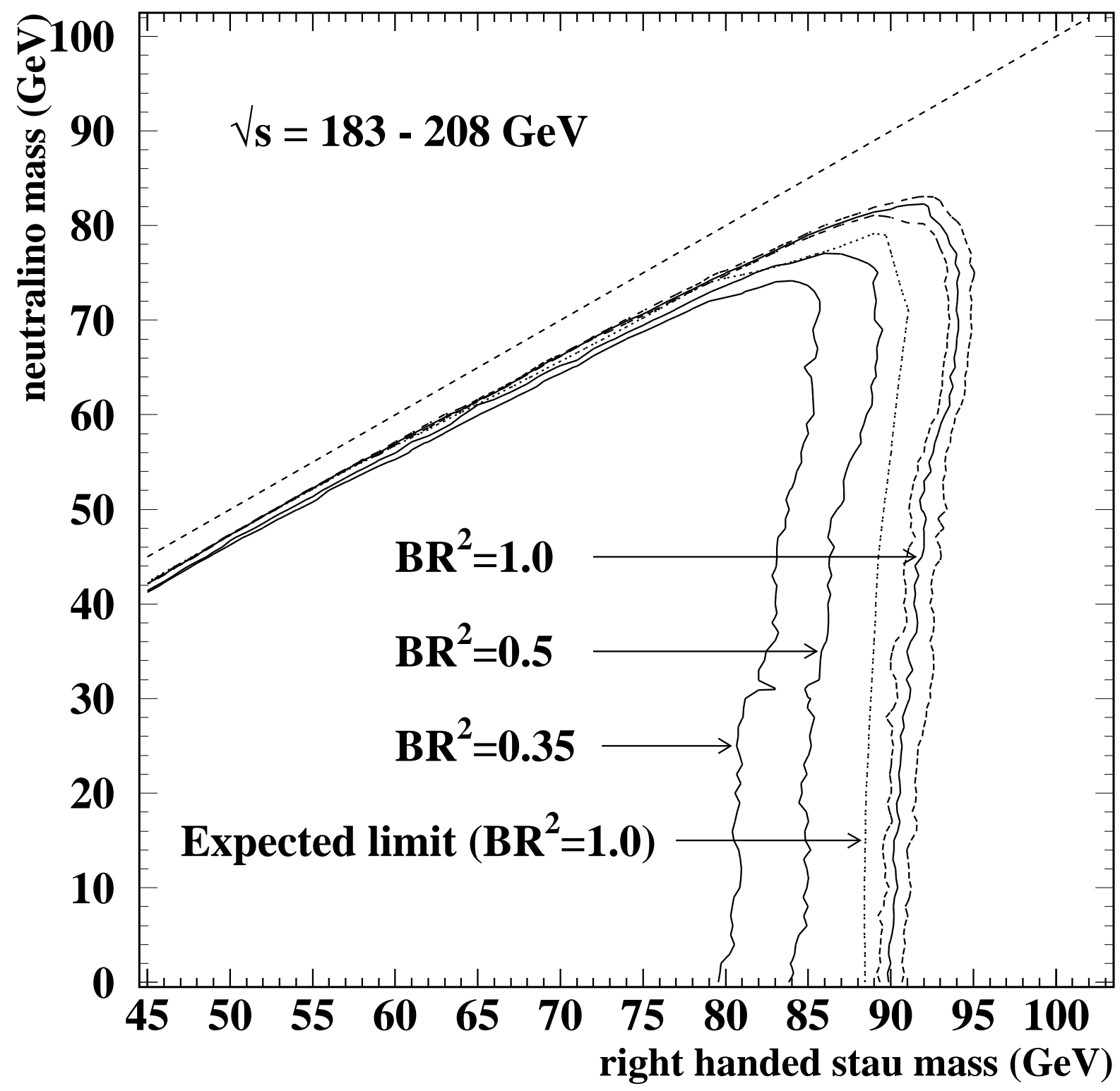

Figure 12: 95\% CL exclusion region for right-handed stau pair production obtained by combining the $\sqrt{s}=183-208 \mathrm{GeV}$ data-sets. The limits are calculated for several values of the branching ratio squared for $\tilde{\tau}_{R}^{ \pm} \rightarrow \tau^{ \pm} \tilde{\chi}_{1}^{0}$. The selection efficiency for $\tilde{\tau}^{+} \tilde{\tau}^{-}$is calculated for the case that the decay $\tilde{\tau}^{-} \rightarrow \tau^{-} \tilde{\chi}_{1}^{0}$ produces unpolarised $\tau^{ \pm}$. Otherwise the limits have no supersymmetry model assumptions. The two broken lines adjacent to the limit for $B R^{2}=1.0$ show the region in which this limit can vary if stau mixing occurs (see text). The kinematically allowed region is shown by the dashed line. The expected limit for $B R^{2}=1.0$, calculated from Monte Carlo alone, is represented by the indicated broken line. 
OPAL

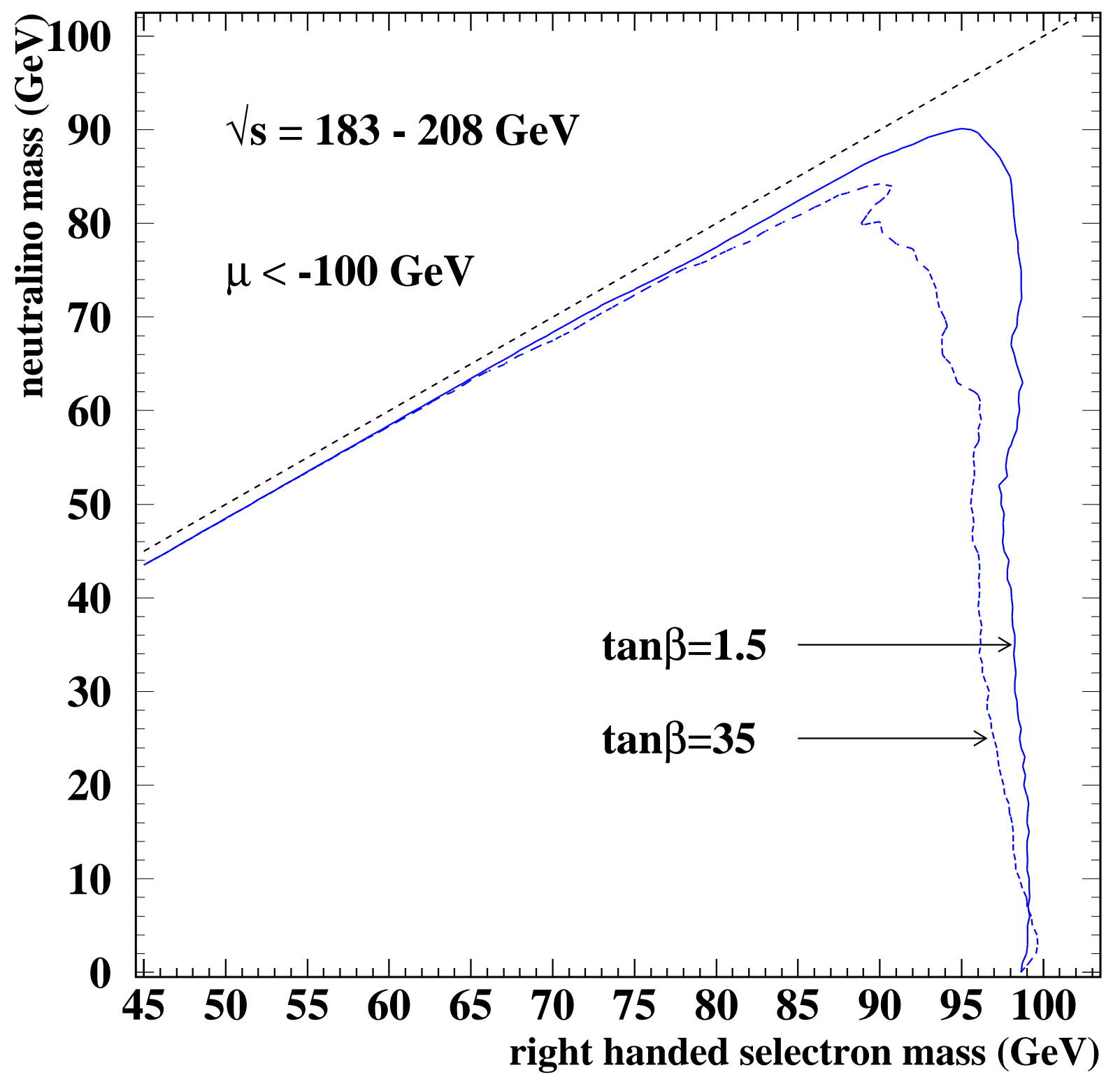

Figure 13: For two values of $\tan \beta$ and $\mu<-100 \mathrm{GeV}, 95 \% \mathrm{CL}$ exclusion regions for righthanded selectron pairs within the MSSM, obtained by combining the $\sqrt{s}=183-208 \mathrm{GeV}$ datasets. The excluded regions are calculated taking into account the predicted branching ratio for $\tilde{\mathrm{e}}_{R}^{ \pm} \rightarrow \mathrm{e}^{ \pm} \tilde{\chi}_{1}^{0}$. The gauge unification relation, $M_{1}=\frac{5}{3} \tan ^{2} \theta_{W} M_{2}$, is assumed in calculating the MSSM cross-sections and branching ratios. The kinematically allowed region lies below the dashed line. 
OPAL

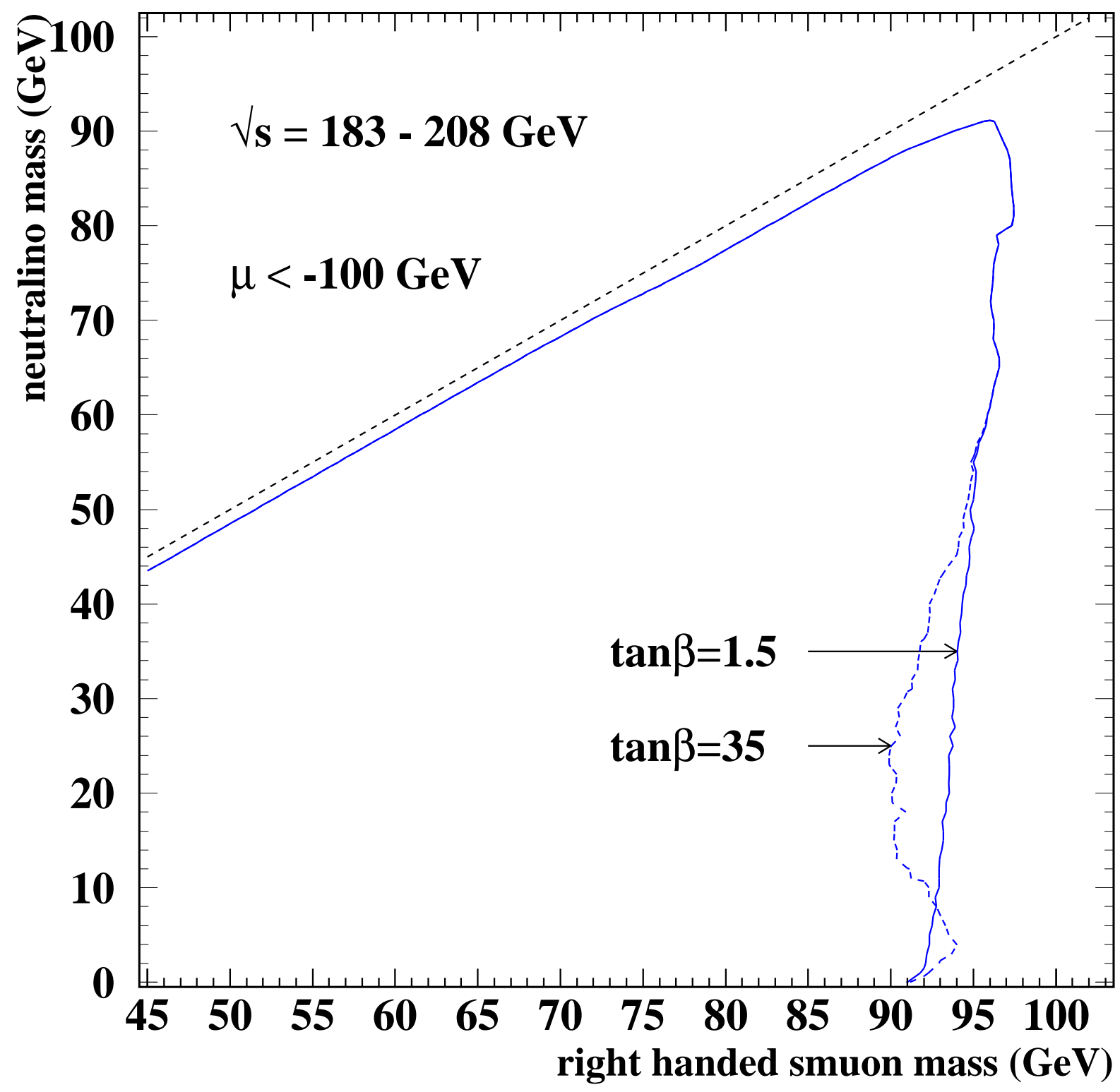

Figure 14: For two values of $\tan \beta$ and $\mu<-100 \mathrm{GeV}$, 95\% CL exclusion regions for righthanded smuon pairs within the MSSM, obtained by combining the $\sqrt{s}=183-208 \mathrm{GeV}$ datasets. The excluded regions are calculated taking into account the predicted branching ratio for $\tilde{\mu}_{R}^{ \pm} \rightarrow \mu^{ \pm} \tilde{\chi}_{1}^{0}$. The gauge unification relation, $M_{1}=\frac{5}{3} \tan ^{2} \theta_{W} M_{2}$, is assumed in calculating the MSSM branching ratios. The kinematically allowed region lies below the dashed line. 


\section{OPAL}

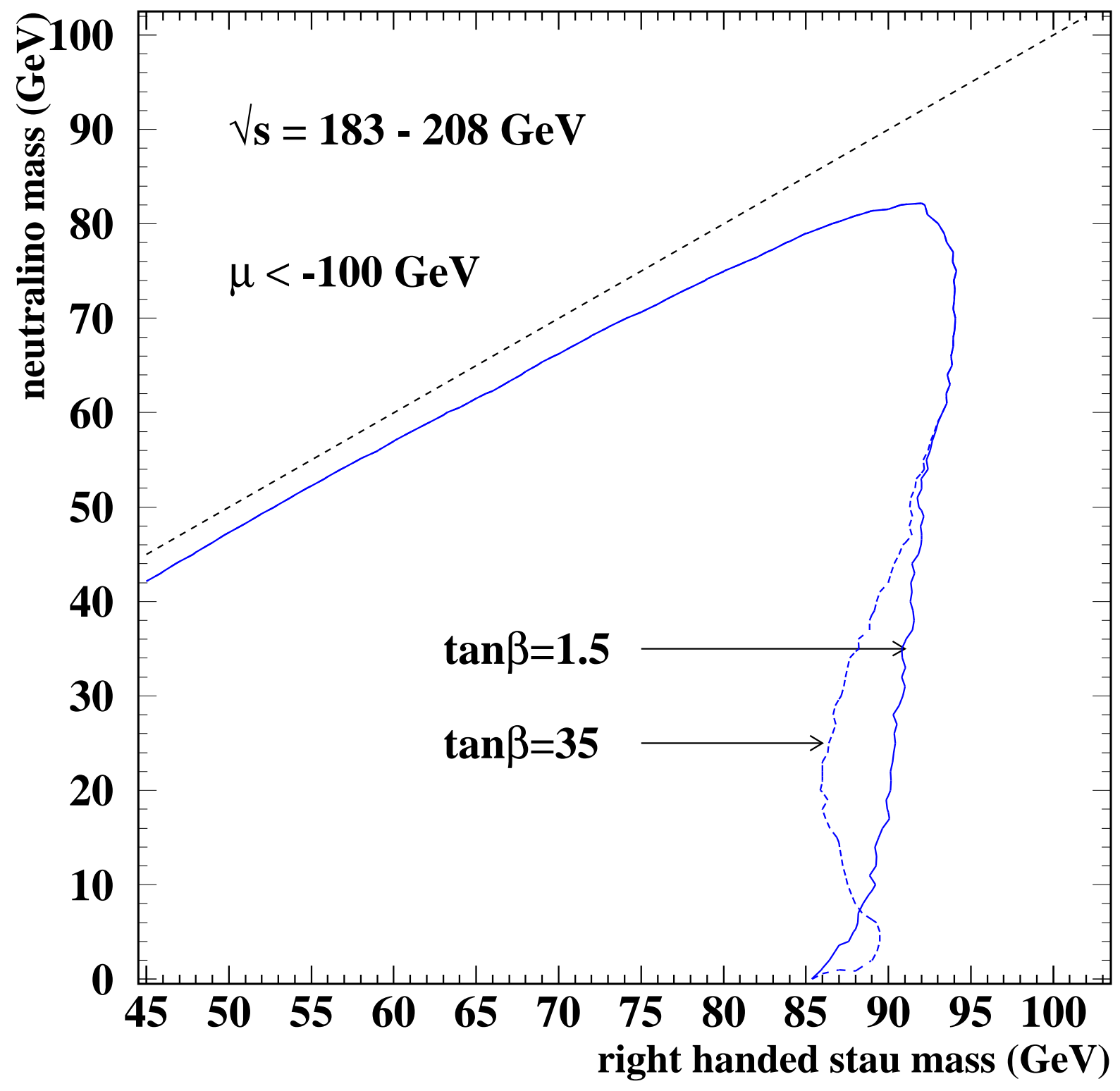

Figure 15: For two values of $\tan \beta$ and $\mu<-100 \mathrm{GeV}$, 95\% CL exclusion regions for righthanded stau pairs within the MSSM, obtained by combining the $\sqrt{s}=183-208$ GeV datasets. The excluded regions are calculated taking into account the predicted branching ratio for $\tilde{\tau}_{R}^{ \pm} \rightarrow \tau^{ \pm} \tilde{\chi}_{1}^{0}$. The gauge unification relation, $M_{1}=\frac{5}{3} \tan ^{2} \theta_{W} M_{2}$, is assumed in calculating the MSSM branching ratios. The selection efficiency for $\tilde{\tau}^{+} \tilde{\tau}^{-}$is calculated for the case that the decay $\tilde{\tau}^{-} \rightarrow \tau^{-} \tilde{\chi}_{1}^{0}$ produces unpolarised $\tau^{ \pm}$. The kinematically allowed region lies below the dashed line. 